This is an Open Access article, distributed under the terms of the Creative Commons Attribution licence (http://creativecommons.org/licenses/by/4.0/), which permits unrestricted re-use, distribution, and reproduction in any medium, provided the original work is properly cited.

doi:10.1017/jfm.2019.714

\title{
Bacterial spinning top
}

\author{
Kenta Ishimoto $\dagger$ \\ Research Institute for Mathematical Sciences, Kyoto University, Kyoto 606-8502, Japan \\ (Received 19 March 2019; revised 16 June 2019; accepted 24 August 2019; \\ first published online 10 October 2019)
}

We have investigated the dynamics of a monotrichous bacteria cell near a wall boundary, taking elastic hook flexibility into consideration. Combining theoretical linear stability analysis and direct numerical computations via the boundary element method, we have found that the elastohydrodynamic coupling between the hook elasticity and cell rotational motion enables a stable vertical spinning behaviour like a low-Reynolds-number spinning top. The forwardly rotated flagellum, which generates the force exertion pushing towards the cell body, typically destabilizes the vertical upright position and leads to a boundary-following motion. In contrast, the backward rotation of the flagellum, generating a force pulling the cell body, contributes to stable upright behaviour in a large range of hook rigidity. Further numerical investigations have demonstrated that the non-spherical geometry of the cell body and boundary adhesive interactions affect the bacterial dynamics, leading to complex behaviours such as horizontal spinning and unstable vertical spinning motions, both of which are experimentally observed in Pseudomonas aeruginosa bacteria. These results highlight the rich diversity of bacterial surface motility emerging from mechanical boundary interactions coupled with the cell swimming and hook flexibility.

Key words: micro-organism dynamics, propulsion, swimming/flying

\section{Introduction}

Bacteria, a major domain of prokaryotic micro-organisms, constitute the bulk of biomass on Earth, and over $90 \%$ of bacteria live on surfaces, where they grow and form architectural structures known as biofilm. The biofilm is not only a dominant form of bacterial life on Earth but also affects human health beneficially and infectiously as it is resistant against chemical and physical stresses (Drescher et al. 2016). The formation of biofilm is initiated when a single swimming cell settles down at a surface, and mechanical interactions between the cell and the surface play a critical role (Persat et al. 2015; Conrad \& Poling-Skutvik 2018).

The bacterial swimming motility is generated by helical filamentous appendages, called flagellar filaments, which are connected to a cell body via flexible hooks. The filament is rotated driven by a molecular motor at the base end of the filament, and the filament-hook-motor complex is called a flagellum (Lauga 2016).

$\dagger$ Email address for correspondence: ishimoto@kurims.kyoto-u.ac.jp 
Recently, de Anda et al. (2017) have observed the detailed dynamics of the Pseudomonas aeruginosa bacteria cell near a substrate, reconstructing the threedimensional cell images through simulation-aided microscopy. $P$. aeruginosa is a polar monotrichous bacterium with a single flagellum connected to one end of an elongated cell body and is a model micro-organism for the study of biofilm formation as well as a major opportunistic human pathogen. De Anda et al. (2017) have reported a variety of flagella-driven motility patterns, including horizontally spinning behaviour and an unstable vertical spinning motion with its flagella-free end continuously attached to the boundary, which they describe in their abstract as 'like [a] low Reynolds number spinning top with an unstable orbit'.

The dynamics of a spinning top is a classic problem in mechanics, but in general predictions of its motion are not trivial due to the nonlinear nature of the Euler equation of the rigid rotation motion and the surface friction force, the latter of which is known to promote a spinning top to stand upright (Braams 1952) and is essential for a vertically spinning egg (Moffatt \& Shimomura 2002; Moffatt, Shimomura \& Branicki 2004). Owing to the small size of the bacterial cell (typical flagellar length $\approx 10 \mu \mathrm{m}$ ), the Reynolds number around a motile bacterium is very small, where the viscous dissipation is dominating; thus a continuous energy input is required to sustain the spinning dynamics. The bacterial motor torque would produce the necessary energy input, but how does the upright spinning motion emerge?

Our understanding of the bacterial behaviour near a wall boundary has progressed in the last decade from theoretical, computational and experimental perspectives (Lauga et al. 2006; Shum, Gaffney \& Smith 2010; Drescher et al. 2011; Lauga 2016; Bianchi, Saglimbeni \& Di Leonardo 2017). The general dynamics of the cell hydrodynamics near a wall boundary is, however, still inadequate to come to any conclusion about the general mechanism of vertical spinning behaviour of the bacterial cell. Vertical spinning motions near a wall boundary have been observed in Thiovulum majus bacteria (Petroff, Wu \& Libchaber 2015), and this mechanism was later examined with the lubrication theory, which shows that bacterial propulsion generates the torque that orients the cell to stand upright quickly $(\approx 0.02 \mathrm{~s})$ (Petroff \& Libchaber 2018). In contrast, Escherichia coli bacteria cells are known to exhibit a boundary-following behaviour (Bianchi et al. 2017), and the same lubrication theory predicts that the time scale of the cell reorientation of the bacteria perpendicular to the surface is much larger than the typical swimming time scale (Lauga et al. 2006). Note that both species are peritrichous bacteria, which possess multiple flagella distributed on the cell surface, but simple arguments based on the lubrication theory provide the opposite conclusions for different bacterial species, emphasizing the necessity of a comprehensive theoretical study of the hydrodynamic interactions among the bacterial cell body and the wall boundary in order to understand the mechanics and stability of a bacterial spinning top.

Compared with the flagellar filament consisting of helical polymers, the short flagellar hook ( $\approx 60 \mathrm{~nm}$ in length) is a flexible structure so that it acts like a universal joint, and can be buckled during the swimming motion (Son, Guasto \& Stocker 2013), generating a rich diversity of bacterial behaviours (Shum \& Gaffney 2012; Nguyen \& Graham 2017, 2018; Jabbarzadeh \& Fu 2018). Riley, Das \& Lauga (2018) considered the hook elasticity for peritrichous bacteria and numerically demonstrated that the elastohydrodynamic instability enables their swimming. They also considered a theoretical model of a sphere with two active rods and local hydrodynamic interactions based on the resistive force theory, and the model successfully predicted the onset of the instability. We thus consider a local hydrodynamic model for physically intuitive 
understandings of the bacterial spinning dynamics, following the stability theory, which has been later extended to more general situations (Ishimoto \& Lauga 2019).

Nonetheless, a direct hydrodynamic computation is essential to resolve the subtle cell-wall hydrodynamic interactions (Spagnolie \& Lauga 2012; Ishimoto \& Gaffney 2013), and thus we also perform detailed numerical simulation based on the boundary element method, which is an established computational methodology in microswimmer hydrodynamics (Pozrikidis 2002; Smith 2018). The primary purpose of this paper is therefore to examine the bacterial behaviour near a wall with consideration of the hook flexibility to understand the bacterial spinning top dynamics, combined with a theoretical stability analysis and hydrodynamic computational simulation.

In de Anda et al. (2017), the authors proposed a simple mathematical model bacterium that consisted of a helical flagellar filament connected to the cell body via a torque spring hook. They considered a cylindrical cell body and hydrodynamic forces that were calculated by a formula for a circular disk in two-dimensional flow (Wakiya 1975) and neglected the non-local hydrodynamic interactions between the cell body, flagellum and boundary. The cylindrical body is anchored to the boundary and only the orientation dynamics has been considered. With this modelling, they demonstrated that a misalignment between the cell body and flagellar orientations could generate the necessary torque for a cell to orient towards the perpendicular configuration. However, their model is insufficient to discuss the stability when the cell body approaches the standing position due to the modelling accuracy of the hydrodynamic interactions. Moreover, the lack of horizontal movement of the cell cannot capture the variety of the flagella-driven motilities such as horizontally spinning motion. Considering the non-trivial hydrodynamic interactions near a wall boundary and the complicated mechanical coupling between the hook flexibility and the entire cell motions, the bacterial behaviours are still unclear when the cell is allowed to translate along the substrate.

Furthermore, in actual biological situations, the cell-surface interaction contains non-hydrodynamic forces, such as an electrostatic repulsion and an adhesive attraction. The details of the cell-surface interactions depend on the cell, surface and physiological solutes (Klein, Clapp \& Dickinson 2003; Chen et al. 2011; Perni, Preedy \& Prokopovich 2014), but here we first focus on pure hydrodynamic interactions, considering a fixed separation distance between the cell surface and the wall boundary. In addition to the complicated cell-surface electrostatic interactions, Pseudomonas utilizes type IV pili, hairy structures on the cell surface, for cell motility and cell adhesion (Maier \& Wong 2015; Conrad \& Poling-Skutvik 2018), which makes the cell-substrate interactions more complicated. In the latter part of this paper, we briefly consider an adhesive interaction, motivated by the nano-spring behaviour of the pili adhesion (Beaussart et al. 2014), modelled by spring bond formation between the cell and wall surfaces, to understand the mechanism underlying the rich behaviours of bacterial motility.

In summary, the hydrodynamic interactions near a wall boundary are, in general, non-trivial, and thus the mechanical stability around the vertical spinning motion has not been analysed even in the rigid hook case. Although the hook flexibility has been suggested to generate the torque that orients the cell to stand vertically by a misalignment between the cell body and flagellar orientations, the whole bacterial dynamics has not been examined when the cell is allowed to move horizontally along the wall boundary. In this paper, motivated by the recent observation of the complicated flagella-driven motility of $P$. aeruginosa near a wall boundary, we will theoretically and numerically investigate the bacterial dynamics near a wall with 


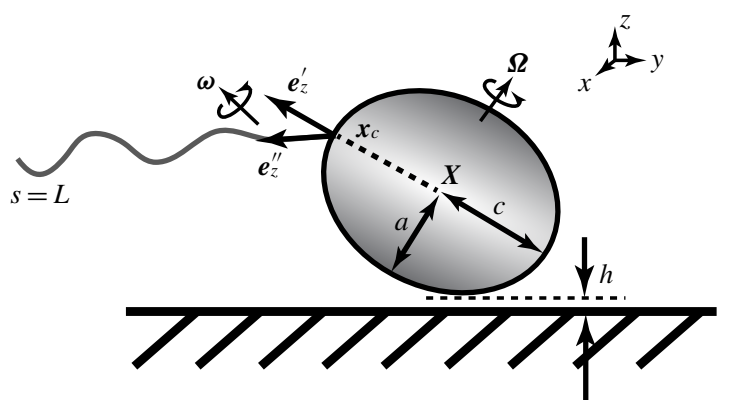

FIGURE 1. Schematic picture of a model bacterium.

consideration of the hook flexibility, focusing on the stability of the vertical spinning motion and the effects of the details on the cell-wall interactions and of the cell body and flagellar morphology.

The contents of this paper are the following. In $\S 2$, we explain the model bacterium and boundary element method to solve the non-local hydrodynamic interactions. In $\S 3$, we formulate a local hydrodynamic interaction theory based on the lubrication theory and the resistive force theory for flagellar propulsion, and theoretically analyse the linear stability around the vertical spinning motion. The following two sections, $\S \S 4$ and 5, are devoted to the results obtained by the numerical simulation. We first consider a spherical cell body to compare the simulation results with the theoretical stability analysis, and then proceed to the non-spherical case and free-swimming simulation, considering the adhesive boundary interaction. In $\S 6$, we summarize the obtained results and discuss the biological implications from those results.

\section{Bacterial hydrodynamics}

\subsection{Model bacterium}

We consider a mono-flagellated bacterial swimmer near an infinite flat wall boundary in a Newtonian fluid of constant viscosity $\mu$. The cell body is assumed to be a spheroid or a spherocylinder of minor semi-axes $a$ and principal axis $c(a \leqslant c)$, including the spherical case. We denote the centre position of the cell body by $\boldsymbol{X}$, and the bacterium is assumed to possess a single flagellum that is connected to one end of the major axis, as schematically illustrated in figure 1.

To describe the bacterial dynamics, we introduce three reference frames (Smith et al. 2009; Ishimoto \& Gaffney 2016): the laboratory frame $\left\{\boldsymbol{e}_{i}\right\}$, the body-fixed frame $\left\{\boldsymbol{e}_{i}^{\prime}\right\}$ and the flagellum-fixed frame $\left\{\boldsymbol{e}_{i}^{\prime \prime}\right\}$ for $i=x, y, z$. The body-fixed frame is obtained by a rotation of the laboratory frame by the rotation matrix $\boldsymbol{B}$, given by $B_{i j}=\boldsymbol{e}_{i} \cdot \boldsymbol{e}_{j}^{\prime}$. The flagellum-fixed frame is obtained by a rotation of the body-fixed frame, and the rotation matrix $\boldsymbol{F}$ is given by $F_{i j}=\boldsymbol{e}_{i}^{\prime} \cdot \boldsymbol{e}_{j}^{\prime \prime}$. The origin of the body-fixed coordinates is set to be the centre of the cell body, and that of the flagellum-fixed coordinates is the connection point of the flagellum and the cell body, $\boldsymbol{x}_{c}$. The swimmer surface $S$ is the union of the cell-body surface $S_{B}$ and flagellum surface $S_{F}$, and we write the position vector $\boldsymbol{x}_{0}$ on the surface $S$ as

$$
\boldsymbol{x}_{0}= \begin{cases}\boldsymbol{X}+\left(\boldsymbol{x}_{0}-\boldsymbol{X}\right), & \boldsymbol{x}_{0} \in S_{B}, \\ \boldsymbol{X}+\left(\boldsymbol{x}_{c}-\boldsymbol{X}\right)+\left(\boldsymbol{x}_{0}-\boldsymbol{x}_{c}\right), & \boldsymbol{x}_{0} \in S_{F}\end{cases}
$$


The relative position vectors $\xi_{B}=\boldsymbol{x}_{0}-\boldsymbol{X}\left(\boldsymbol{x}_{0} \in S_{B}\right)$ and $\boldsymbol{\xi}_{F}=\boldsymbol{x}_{0}-\boldsymbol{x}_{c}\left(\boldsymbol{x}_{0} \in S_{F}\right)$ can be obtained by the rotations,

$$
\boldsymbol{\xi}_{B}=\boldsymbol{B} \cdot \hat{\boldsymbol{\xi}}_{B}, \quad \boldsymbol{\xi}_{F}=\boldsymbol{B} \cdot \boldsymbol{F} \cdot \hat{\boldsymbol{\xi}}_{F},
$$

where $\hat{\xi}_{B}$ and $\hat{\xi}_{F}$ are the components measured in the body-fixed and flagellum-fixed frames, respectively. The vectors are therefore constant in time. We then take the Lagrangian time derivative for the position vector, $\boldsymbol{x}_{0}$, to derive the expressions of the velocity. Noting that only the rotation matrices are functions of time, we obtain

$$
\boldsymbol{v}\left(\boldsymbol{x}_{0}\right)= \begin{cases}\boldsymbol{U}+\boldsymbol{\Omega} \times\left(\boldsymbol{x}_{0}-\boldsymbol{X}\right), & \boldsymbol{x}_{0} \in S_{B}, \\ \boldsymbol{U}+\boldsymbol{\Omega} \times\left(\boldsymbol{x}_{c}-\boldsymbol{X}\right)+(\boldsymbol{B} \cdot \boldsymbol{\omega}) \times\left(\boldsymbol{x}_{0}-\boldsymbol{x}_{c}\right), & \boldsymbol{x}_{0} \in S_{F},\end{cases}
$$

where the linear and angular velocities are defined as $\dot{\boldsymbol{X}}=\boldsymbol{U}, \dot{\boldsymbol{B}}=\boldsymbol{\Omega} \times \boldsymbol{B}$ and $\dot{\boldsymbol{F}}=$ $\boldsymbol{\omega} \times \boldsymbol{F}$, with the dots denoting the time derivatives.

We assume a cylindrical flagellum of radius $d$, and, following Higdon (1979) and Shum et al. (2010), consider a helical waveform of the flagellum whose centreline is given in terms of the flagellum-fixed coordinates $(\hat{x}(\hat{z}, t), \hat{y}(\hat{z}, t), \hat{z})$ by

$$
\begin{aligned}
& \hat{x}=A\left(1-\mathrm{e}^{-k_{E^{2}}^{2} z^{2}}\right) \cos (k \hat{z}), \\
& \hat{y}=-A\left(1-\mathrm{e}^{-k_{E}^{2} z^{2}}\right) \sin (k \hat{z}) .
\end{aligned}
$$

Here, $A$ is the amplitude of the helix, $k$ is the wavenumber and the parameter $k_{E}$ governs the scale of the initial increase in the flagellar envelope. The functions (2.4) and (2.5) are defined within the range of $\hat{z} \in\left[0, L_{e}\right]$, where the coordinate of the flagellar distal end, $L_{e}>0$, is implicitly defined by

$$
L=\int_{0}^{L_{e}} \sqrt{1+\left(\frac{\mathrm{d} \hat{x}}{\mathrm{~d} \hat{z}}\right)^{2}+\left(\frac{\mathrm{d} \hat{y}}{\mathrm{~d} \hat{z}}\right)^{2}} \mathrm{~d} \hat{z} .
$$

\subsection{Mechanical equations}

Owing to the small size of the bacterial swimmer, one can neglect the inertia effects and thus the fluid motions obey the Stokes equation

$$
\nabla p=\mu \Delta \boldsymbol{u},
$$

where $\boldsymbol{u}$ is the fluid velocity and $p$ is the pressure field. The fluid velocity can be represented by the single-layer boundary integral form (Pozrikidis 2002; Shum \& Gaffney 2012)

$$
\boldsymbol{u}(\boldsymbol{x})=-\int_{S\left(x_{0}\right)} \boldsymbol{G}\left(\boldsymbol{x}, \boldsymbol{x}_{0}\right) \cdot \boldsymbol{q}\left(\boldsymbol{x}_{0}\right) \mathrm{d} S\left(\boldsymbol{x}_{0}\right) .
$$

The integral kernel, $G$, is the blakelet (Blake 1971), which satisfies the zero velocity condition on the wall surface, $z=0$, and the integral is performed over the entire cell surface, $S$. Since the swimmer inertia is also negligible at low Reynolds number, the single-layer potential $\boldsymbol{q}\left(\boldsymbol{x}_{0}\right)$ satisfies the force and torque balance equations, which read

$$
\int_{S\left(x_{0}\right)}\left[\boldsymbol{q}+\boldsymbol{f}_{e x t}\right] \mathrm{d} S\left(\boldsymbol{x}_{0}\right)=\int_{S\left(x_{0}\right)}\left(\boldsymbol{x}_{0}-\boldsymbol{X}\right) \times\left[\boldsymbol{q}\left(\boldsymbol{x}_{0}\right)+\boldsymbol{f}_{\text {ext }}\right] \mathrm{d} S\left(\boldsymbol{x}_{0}\right)=\mathbf{0},
$$


where $f_{\text {ext }}$ is the external force and will be considered for the cell-wall interactions. The no-slip boundary condition on the swimmer surface, $S$, is given by $\boldsymbol{u}\left(\boldsymbol{x}_{0}\right)=\boldsymbol{v}\left(\boldsymbol{x}_{0}\right)$ and obtained from equations (2.3) and (2.8).

We consider a flexible hook that connects the cell body and the flagellum, modelling it as a torque spring following previous studies (Bennett et al. 2016; de Anda et al. 2017; Nguyen \& Graham 2017; Riley et al. 2018). At the rigid limit of the hook, the flagellum is assumed to be connected normal to the body, or $\boldsymbol{e}_{z}^{\prime}=\boldsymbol{e}_{z}^{\prime \prime}$. When the hook is flexible, the relative angle between the two vectors, $\boldsymbol{e}_{z}^{\prime}$ and $\boldsymbol{e}_{z}^{\prime \prime}$, can take a non-zero value, but we assume that it eventually relaxes to zero by the hook torque spring. Let $f(\varphi)$ be the function that presents the magnitude of the recovery torque, where the angle $\varphi$ is the relative angle, $\varphi=\sin ^{-1}\left|\boldsymbol{e}_{z}^{\prime} \times \boldsymbol{e}_{z}^{\prime \prime}\right|$. The bending torque due to the hook flexibility is therefore given by $\boldsymbol{M}_{\text {bend }}=-f(\varphi) \boldsymbol{e}_{\perp}^{\prime}$, with the unit vector $\boldsymbol{e}_{\perp}^{\prime}$ given by $\boldsymbol{e}_{\perp}^{\prime}=\boldsymbol{e}_{z}^{\prime} \times \boldsymbol{e}_{z}^{\prime \prime} /\left|\boldsymbol{e}_{z}^{\prime} \times \boldsymbol{e}_{z}^{\prime \prime}\right|$. With a positive constant $\kappa$, we assume $f(\varphi)=\kappa \tan \varphi$, and thus the bending torque behaves as a linear torque spring with spring constant $\kappa$ if the angle is small and diverges as the angle approaches $\pi / 2$, preventing the flagellum from penetrating to the cell body.

The flagellum is rotated by motor torque at the flagellar base, $\boldsymbol{M}_{m o t}$. In this study we assume a constant motor torque applied towards the normal to the cell-body surface, $\boldsymbol{M}_{m o t}=\tau \boldsymbol{e}_{z}^{\prime}$ (Shum \& Gaffney 2012; Shimogonya et al. 2015; Jabbarzadeh \& Fu 2018).

Finally, we have the torque balance equation around the connection point $\boldsymbol{x}_{c}$ :

$$
\int_{S_{F}\left(x_{0}\right)}\left(\boldsymbol{x}_{0}-\boldsymbol{x}_{c}\right) \times \boldsymbol{q}\left(\boldsymbol{x}_{0}\right) \mathrm{d} S_{F}\left(\boldsymbol{x}_{0}\right)+\boldsymbol{M}_{\text {bend }}+\boldsymbol{M}_{m o t}=\mathbf{0},
$$

noting that the surface integral should be computed only over the flagellar surface.

\subsection{Wall conditions}

In this study, we first fix the value of the separation distance between the wall boundary and the cell-body surface, $h$. This assumption can be biologically interpreted by an attraction to a minimum of the surface potential, which ranges over the orders of 10-100 nm (Chen et al. 2011). We define the contact point, $\boldsymbol{X}_{p}$, as the point on the cell body closest to the wall boundary, and let the angle of the cell-body inclination be $\theta=\cos ^{-1}\left(\boldsymbol{e}_{z}^{\prime} \cdot \boldsymbol{e}_{z}\right) \in[0, \pi]$ and consider the distance between the body centre and the wall boundary, $H(\theta)$, given by

$$
H(\theta)=h+ \begin{cases}\sqrt{a^{2} \sin ^{2} \theta+c^{2} \cos ^{2} \theta} & \text { (spheroid), } \\ a+(c-a)|\cos \theta| & \text { (spherocylinder). }\end{cases}
$$

The $z$ component of the swimming velocity, $U_{z}$, is geometrically determined by the constraint of constant separation, and this can be simply obtained (Moffatt \& Shimomura 2002; Moffatt et al. 2004) by

$$
U_{z}=\frac{\mathrm{d} H}{\mathrm{~d} \theta} \frac{\mathrm{d} \theta}{\mathrm{d} t}=\left(\frac{\mathrm{d} H}{\mathrm{~d} \theta}\right) \boldsymbol{e}_{\perp} \cdot \boldsymbol{\Omega},
$$

where $\boldsymbol{e}_{\perp}$ is introduced by $\boldsymbol{e}_{\perp}=\boldsymbol{e}_{z} \times \boldsymbol{e}_{z}^{\prime} /\left|\boldsymbol{e}_{z} \times \boldsymbol{e}_{z}^{\prime}\right|$. Note that the vector $\boldsymbol{e}_{\perp}$ becomes singular around $\theta \approx 0$ but the singular factor can be absorbed into the derivatives term in (2.12). From direct calculations, we have

$$
U_{z}= \begin{cases}-\frac{\left(c^{2}-a^{2}\right) \cos \theta}{H-h}\left(\boldsymbol{e}_{z} \times \boldsymbol{e}_{z}^{\prime}\right) \cdot \boldsymbol{\Omega} & \text { (spheroid), } \\ -(c-a) \operatorname{sgn}(\cos \theta)\left(\boldsymbol{e}_{z} \times \boldsymbol{e}_{z}^{\prime}\right) \cdot \boldsymbol{\Omega} & \text { (spherocylinder) } .\end{cases}
$$


The velocity constraint relation (2.13) becomes discontinuous at $\theta=\pi / 2$ for the spherocylindrical cell-body case, and we will terminate the numerical computation when the inclination angle approaches $\theta \approx \pi / 2$.

In the constant-separation situation, there are no boundary-related external forces in the horizontal direction. We thus set $f_{\text {ext }}=\left(0,0, f_{e x t}\right)$ in the force and torque balance equations (2.9), and only hydrodynamic interactions are considered. In the $z$ direction, we have imposed zero velocity condition, $U_{z}=0$, instead of the force balance equations.

Later in this paper, we will consider a fully three-dimensional numerical simulation, in which the cell is allowed to move in the perpendicular direction as well. At the very vicinity of the wall surface, the electrostatic potential is repulsive, and we assume the following short-range repulsive wall interactions per unit area of the cell surface (Ishikawa \& Pedley 2007; Spagnolie \& Lauga 2012; Ishimoto \& Gaffney 2016):

$$
\boldsymbol{f}_{\text {rep }}(\boldsymbol{x})=g \frac{\mathrm{e}^{-z / \lambda}}{1-\mathrm{e}^{-z / \lambda}} \boldsymbol{e}_{z},
$$

where $g$ and $\lambda$ are the strength and decay length of the wall repulsive force, respectively. The force decay length, therefore, provides the characteristic length of the minimum separation distance.

We also consider an adhesive attractive interaction between the cell-body surface and the wall, motivated by the nano-spring property of the pili adhesion of P. aeruginosa (Beaussart et al. 2014). We simply model the adhesive interaction by an elastic bond, following the receptor-ligand binding between the sperm and the wall boundary (Ishimoto \& Gaffney 2016). Elastic bonds are formed at each cell-body surface, $\boldsymbol{x}$, once the distance from the wall is closer than $R_{o n}$, with the location of the bond $\boldsymbol{X}_{a d}$ given by $\boldsymbol{X}_{a d}=\boldsymbol{x} \cdot\left(\mathbf{1}-\boldsymbol{e}_{z} \boldsymbol{e}_{z}\right)+R_{a d} \boldsymbol{e}_{z}$. The adhesive force is then assumed to be that of the linear spring,

$$
\boldsymbol{f}_{a d}(\boldsymbol{x})=-k\left(\boldsymbol{x}-\boldsymbol{X}_{a d}\right),
$$

where $k$ is the spring constant of the bond. The adhesive bond is, however, broken and removed when the bond length exceeds the length $R_{\text {off }}$. Owing to the wall-associated forces, we set $\boldsymbol{f}_{\text {ext }}=\boldsymbol{f}_{\text {rep }}+\boldsymbol{f}_{\text {ad }}$ in the force and torque balance equations (2.9).

\subsection{Numerical methods}

We solve the above set of equations via the boundary element method, following our previous studies (Ishimoto \& Gaffney 2014, 2017). We discretize the cell-body surface using finer meshes near a contact point to resolve the hydrodynamic interactions efficiently (figure $2 b$ ). We have discretized the cell body by 512 meshes and the flagellum by 186 meshes. The validation of the numerics is obtained by comparison with the asymptotic analysis of a sphere translated by a constant force (Goldmann, Cox \& Brenner 1967), but the numerics loses its accuracy when the separation distance is too small $(h / a \lesssim 0.003)$. We also found that the use of half mesh size (fourfold total number of meshes) does not improve the resolution for the finer separation case. We therefore use the above number of meshes and only consider the separation distance with $h / a>0.003$. With $N$ being the total number of the mesh elements on the swimmer surface, the equations can be reduced to a linear system with $3 N+9$ degrees, and be solved instantaneously with respect to the 
(a)

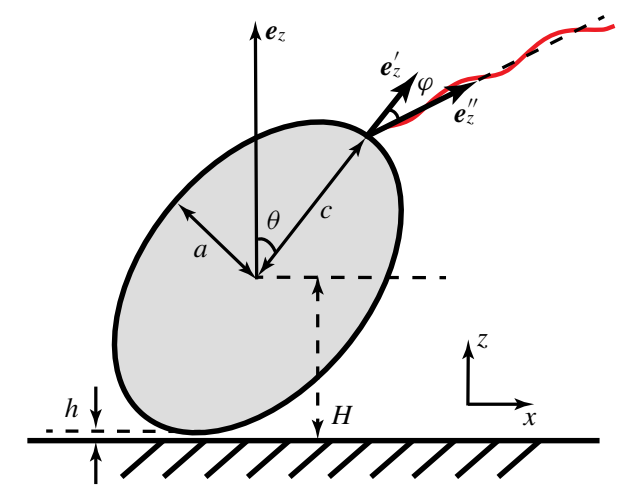

(b)

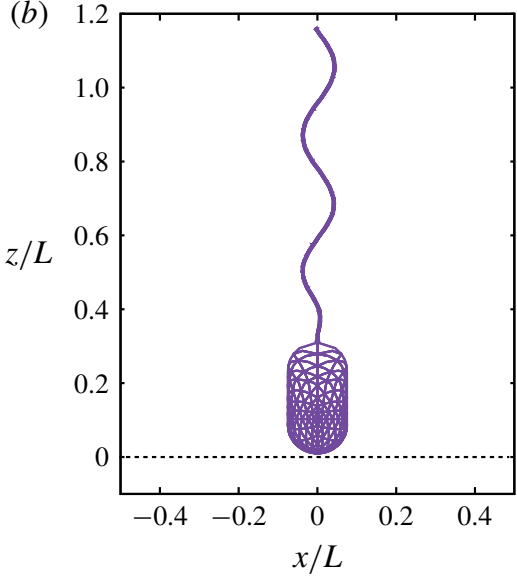

FIGURE 2. (Colour online) (a) Schematic of a model bacterium with a spheroidal cell body. (b) Numerical meshes on a model bacterium surface with a spherocylindrical cell body.

$\begin{array}{lccc}\text { Parameter } & \text { Interpretation } & \text { Flagellum 1 } & \text { Flagellum 2 } \\ d / L & \text { Flagellar radius } & 0.03 & 0.03 \\ A / L & \text { Radius of flagellar helix } & 0.04 & 0.0709 \\ k_{E} L & \text { Smoothing parameter } & 10 & 10 \\ k L /(2 \pi) & \text { Flagellar wavenumber } & 2.7 & 1.953\end{array}$

TABLE 1. List of the parameters of the model flagellum. As a model morphology of $P$. aeruginosa flagellum, we employ the parameter set of flagellum 1, and the parameters of flagellum 2 are used for a parameter robustness analysis.

unknown functions $\boldsymbol{q}, \boldsymbol{U}, \boldsymbol{\Omega}$ and $\boldsymbol{\omega}$. The time evolutions of the swimmer dynamics are implemented via the Heun method.

In the simulation, we use a set of parameters representing a typical morphology of $P$. aeruginosa flagellum (Dasgupta, Arora \& Ramphal 2004) (see flagellum 1 of table 1). The parameters listed under flagellum 2 in table 1 correspond to a bacterial flagellum with an optimal power efficiency obtained by Shum et al. (2010) and used in our previous study (Ishimoto \& Gaffney 2017). This parameter set will be used to check the robustness to the flagellar geometry and will be briefly considered in $\S 4.1$.

We readily find characteristic scales for the length and the force, which are the flagellar length $L=1$ and fluid viscosity $\mu=1$, respectively. The typical physical values of these unit scales are $L=10 \mu \mathrm{m}$ and $\mu=1 \mathrm{mPa}$ s. The characteristic time scale of this system should be determined by the amount of the motor torque, which is estimated as $\approx 2 \times 10^{-18} \mathrm{~N} \mathrm{~m}$ (de Anda et al. 2017). In this study, however, we will explore various sizes of cell body, and the time scale of the emerged swimming dynamics depends on cell geometry. Thus, except in $\S 3.2$, we use $T=0.02 \mathrm{~s}$ as the physical unit of the time scale for convenience. In the numerical simulation, we non-dimensionalize the physical variables with the unit of $L=\mu=T=1$. 


\section{Local interaction theory}

\subsection{Theoretical formulations}

In this section, we approximate the flagellar dynamics by neglecting non-local hydrodynamic interactions, following the theoretical formulation by Ishimoto \& Lauga (2019), in which they study elastohydrodynamic instability induced by the flexible hook for a multi-flagellated spherical bacterial cell in the absence of external boundaries.

We introduce the hydrodynamic drag and torque on the cell body, $\boldsymbol{F}_{b o d y}$ and $\boldsymbol{M}_{b o d y}$, in the form of the linear relation

$$
\left(\begin{array}{l}
\boldsymbol{F}_{\text {body }} \\
\boldsymbol{M}_{\text {body }}
\end{array}\right)=\left(\begin{array}{ll}
\boldsymbol{C}_{T T} & \boldsymbol{C}_{T R} \\
\boldsymbol{C}_{R T} & \boldsymbol{C}_{R R}
\end{array}\right)\left(\begin{array}{c}
\boldsymbol{U} \\
\boldsymbol{\Omega}
\end{array}\right)
$$

The resistive matrix in (3.1) is diagonal for an axisymmetric cell body considered in this study if no external wall boundaries are considered. However, when the cell body is located near a wall boundary, the expressions of the resistive matrix are nondiagonal and the components are given as a function of the separation, $h$. In this section, we only consider a spherical cell body and employ the asymptotic expression derived from the lubrication theory for a sphere in the vicinity of a wall boundary (Goldmann et al. 1967; Lauga et al. 2006). The detailed expressions of the resistive matrix are provided in appendix A.

Similarly to $\S 2.2$, we consider the force and torque balance equations for the whole cell and the flagellum, neglecting the non-local hydrodynamic interactions using the resistive force theory (Lauga \& Powers 2009). We assume that the flagellum is a slender one-dimensional filament, and the flagellar shape is then determined by its tangent vector $\boldsymbol{t}(s)(s \in[0, L])$. From the resistive force theory, the hydrodynamic force on a segment of the flagellum, $\mathrm{d} \boldsymbol{F}(s)$, is linearly related to the local velocity, $\boldsymbol{v}$ in (2.3), as

$$
\mathrm{d} \boldsymbol{F}(s)=\left[C_{t} \boldsymbol{t t}+C_{n}(\mathbf{1}-\boldsymbol{t t})\right] \cdot \boldsymbol{v}(s) \mathrm{d} s,
$$

where $C_{t}$ and $C_{n}$ are negative drag coefficients depending on the flagellar slenderness parameter. Introducing the position vector of the centreline of the flagellum relative to flagellar connecting point $\boldsymbol{x}_{c}$ as

$$
\boldsymbol{\xi}(s)=\int_{0}^{s} \boldsymbol{t}\left(s^{\prime}\right) \mathrm{d} s^{\prime},
$$

we derive the hydrodynamic force on the flagellum, $\boldsymbol{F}$, denoting the drag coefficient tensor in (3.2) by $\boldsymbol{C}$ :

$$
\boldsymbol{F}=\left[\int_{0}^{L} \boldsymbol{C} \mathrm{d} s\right] \cdot \boldsymbol{U}+\left[\int_{0}^{L} \boldsymbol{C} \cdot \boldsymbol{A} \mathrm{d} s\right] \cdot \boldsymbol{\Omega}+\left[\int_{0}^{L} \boldsymbol{C} \cdot \tilde{\boldsymbol{A}} \mathrm{d} s\right] \cdot \boldsymbol{B} \cdot \boldsymbol{\omega} .
$$

Here, we have introduced skew matrices, $\boldsymbol{A}_{i}$ and $\tilde{\boldsymbol{A}}_{i}$, whose components are, respectively, given by

$$
[\boldsymbol{A}]_{i j}=\varepsilon_{i j k}\left(\left(\boldsymbol{x}_{c}-\boldsymbol{X}\right)_{k}+\xi_{k}\right) \quad \text { and } \quad[\tilde{\boldsymbol{A}}]_{i j}=\varepsilon_{i j k} \xi_{k},
$$

where $\varepsilon_{i j k}$ is the Levi-Civita symbol. The force balance equations are obtained by summing up the forces:

$$
\boldsymbol{F}_{\text {body }}+\boldsymbol{F}=\mathbf{0} .
$$


Similarly to the above arguments, we then proceed to the torque balance around the centre of the cell body for an entire cell. We again neglect hydrodynamic interactions between the cell and flagella, and consider hydrodynamic drag on the flagella using the resistive force theory. The torque on a segment of a flagellum around the cellbody centre is given by $\mathrm{d} \boldsymbol{M}=\left(\boldsymbol{\xi}+\left(\boldsymbol{x}_{c}-\boldsymbol{X}\right)\right) \times \mathrm{d} \boldsymbol{F}$, from which we obtain the torque expression by integrating $\mathrm{d} \boldsymbol{M}$ over the flagellum,

$$
\boldsymbol{M}=\left[\int_{0}^{L} \boldsymbol{A}^{\mathrm{T}} \cdot \boldsymbol{C} \mathrm{d} s\right] \cdot \boldsymbol{U}+\left[\int_{0}^{L} \boldsymbol{A}^{\mathrm{T}} \cdot \boldsymbol{C} \cdot \boldsymbol{A} \mathrm{d} s\right] \cdot \boldsymbol{\Omega}+\left[\int_{0}^{L} \boldsymbol{A}^{\mathrm{T}} \cdot \boldsymbol{C} \cdot \tilde{\boldsymbol{A}} \mathrm{d} s\right] \cdot \boldsymbol{B} \cdot \boldsymbol{\omega},
$$

where the superscript $\mathrm{T}$ indicates the transpose of a matrix. We thus have a torque balance equation for the whole cell,

$$
\boldsymbol{M}_{\text {body }}+\boldsymbol{M}=\mathbf{0} .
$$

Lastly, we consider the torque balance relation for the flagellum around the connection of the flagellum and the cell body. Incorporating hydrodynamic torque and elastic spring torque, we have the torque acting on a segment of a flagellum, which is given by $\mathrm{d} \tilde{\boldsymbol{M}}=\boldsymbol{\xi} \times \mathrm{d} \boldsymbol{F}$. Integration over the flagellum yields the hydrodynamic torque,

$$
\tilde{\boldsymbol{M}}=\left[\int_{0}^{L} \tilde{\boldsymbol{A}}^{\mathrm{T}} \cdot \boldsymbol{C} \mathrm{d} s\right] \cdot \boldsymbol{U}+\left[\int_{0}^{L} \tilde{\boldsymbol{A}}^{\mathrm{T}} \cdot \boldsymbol{C} \cdot \boldsymbol{A} \mathrm{d} s\right] \cdot \boldsymbol{\Omega}+\left[\int_{0}^{L} \tilde{\boldsymbol{A}}^{\mathrm{T}} \cdot \boldsymbol{C} \cdot \tilde{\boldsymbol{A}} \mathrm{d} s\right] \cdot \boldsymbol{B} \cdot \boldsymbol{\omega} .
$$

We then have the torque balance equations for the flagellum,

$$
\tilde{\boldsymbol{M}}+\boldsymbol{M}_{\text {elast }}+\boldsymbol{M}_{m o t}=\mathbf{0},
$$

where $\boldsymbol{M}_{m o t}$ is the torque applied at a bacterium motor. In the current problem, the magnitude of the motor torque is assumed to be constant as discussed in the previous section.

The above set of governing equations (3.4)-(3.10) can be summarized into the following matrix form:

$$
\left(\begin{array}{lll}
\boldsymbol{K}_{T T} & \boldsymbol{K}_{T R} & \boldsymbol{K}_{T F} \\
\boldsymbol{K}_{R T} & \boldsymbol{K}_{R R} & \boldsymbol{K}_{R F} \\
\boldsymbol{K}_{F T} & \boldsymbol{K}_{F R} & \boldsymbol{K}_{F F}
\end{array}\right)\left(\begin{array}{c}
\boldsymbol{U} \\
\boldsymbol{\Omega} \\
\boldsymbol{B} \cdot \boldsymbol{\omega}
\end{array}\right)=\left(\begin{array}{c}
\mathbf{0} \\
\mathbf{0} \\
-\boldsymbol{M}_{\text {elast }}-\boldsymbol{M}_{\text {motor }}
\end{array}\right),
$$

where the matrices on the left-hand side read

$$
\left(\begin{array}{ccc}
\boldsymbol{C}_{T T}+\int_{0}^{L} \boldsymbol{C} \mathrm{d} s & \boldsymbol{C}_{T R}+\int_{0}^{L} \boldsymbol{C} \cdot \boldsymbol{A} \mathrm{d} s & \int_{0}^{L} \boldsymbol{C} \cdot \tilde{\boldsymbol{A}} \mathrm{d} s \\
\boldsymbol{C}_{R T}+\int_{0}^{L} \boldsymbol{A}^{\mathrm{T}} \cdot \boldsymbol{C} \mathrm{d} s & \boldsymbol{C}_{R R}+\int_{0}^{L} \boldsymbol{A}^{\mathrm{T}} \cdot \boldsymbol{C} \cdot \boldsymbol{A} \mathrm{d} s & \int_{0}^{L} \boldsymbol{A}^{\mathrm{T}} \cdot \boldsymbol{C} \cdot \tilde{\boldsymbol{A}} \mathrm{d} s \\
\int_{0}^{L} \tilde{\boldsymbol{A}}^{\mathrm{T}} \cdot \boldsymbol{C} \mathrm{d} s & \int_{0}^{L} \tilde{\boldsymbol{A}}^{\mathrm{T}} \cdot \boldsymbol{C} \cdot \boldsymbol{A} \mathrm{d} s & \int_{0}^{L} \tilde{\boldsymbol{A}}^{\mathrm{T}} \cdot \boldsymbol{C} \cdot \tilde{\boldsymbol{A}} \mathrm{d} s
\end{array}\right) .
$$


(a)

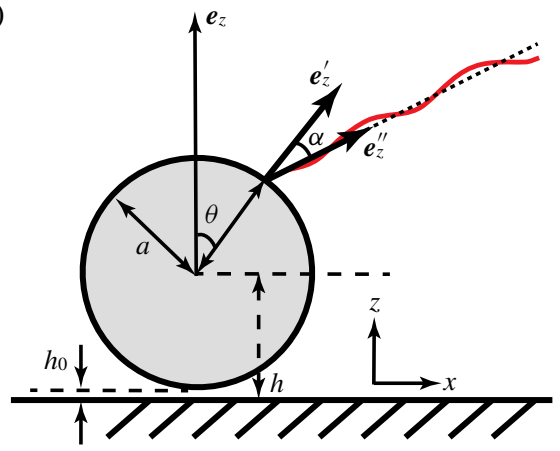

(b)

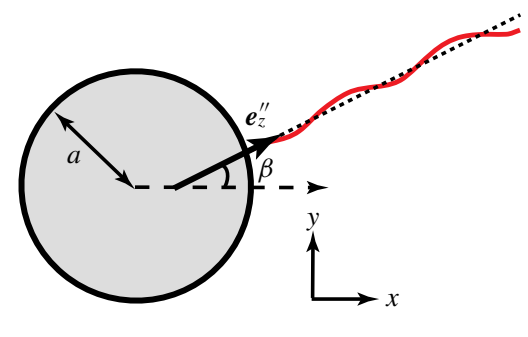

FIgURE 3. (Colour online) Schematic of the model bacterial cell. The flagellum is indicated in red. (a) Projection onto the $x-z$ plane. The angle $\alpha$ is the relative angle between $\boldsymbol{e}_{z}^{\prime}$ and $\boldsymbol{e}_{z}^{\prime \prime}$, measured in the $x-z$ plane. (b) Projection onto the $x-y$ plane. The angle $\beta$ is measured in the $x-y$ plane.

\subsection{Linear stability}

We further simplify the bacterial model to perform linear stability analysis around the upright spinning motion. The typical flagellar rotation is sufficiently rapid compared with the time scale of the flagellar bending, and we approximate the flagellar propulsion by its time-averaged contribution (Lauga et al. 2006). It then follows that the flagellar propulsion is axisymmetric around the flagellar long axis. Only in this section, we use the units of $a=\mu=\tau=1$ for simplicity of mathematical expressions. In the context of the upright spinning motion of a bacterium with a spherical cell body, the emerged time scale is the rotation velocity of the cell body, and this is proportional to $\mu a^{3} / \tau$. In other words, we non-dimensionalize the system using this time scale.

Following Ishimoto \& Lauga (2019), we introduce $3 \times 3$ matrices,

$$
\boldsymbol{K}_{C}=\int_{0}^{L} \boldsymbol{C} \mathrm{d} s, \quad \boldsymbol{K}_{T F}=\int_{0}^{L} \boldsymbol{C} \cdot \tilde{\boldsymbol{A}} \mathrm{d} s, \quad \boldsymbol{K}_{F F}=\int_{0}^{L} \tilde{\boldsymbol{A}}^{\mathrm{T}} \cdot \boldsymbol{C} \cdot \tilde{\boldsymbol{A}} \mathrm{d} s
$$

and the axisymmetric flagellar propulsion enables us to simplify the expressions as

$$
\boldsymbol{K}_{C}^{(0)}=\left(\begin{array}{ccc}
k_{C} & 0 & 0 \\
0 & k_{C} & 0 \\
0 & 0 & K_{C}
\end{array}\right), \quad \boldsymbol{K}_{T F}^{(0)}=\left(\begin{array}{ccc}
k_{D} & k_{T} & 0 \\
-k_{T} & k_{D} & 0 \\
0 & 0 & K_{T}
\end{array}\right), \quad \boldsymbol{K}_{F F}^{(0)}=\left(\begin{array}{ccc}
k_{F} & 0 & 0 \\
0 & k_{F} & 0 \\
0 & 0 & K_{F}
\end{array}\right),
$$

where superscript ' $(0)$ ' indicates the expressions in the flagellum-fixed frame so that the expression in the laboratory frame is transformed by $\boldsymbol{K}_{C}=[\boldsymbol{B} \cdot \boldsymbol{F}] \cdot \boldsymbol{K}_{C}^{(0)} \cdot[\boldsymbol{B} \cdot \boldsymbol{F}]^{-1}$ for instance. The symmetric nature of $\boldsymbol{K}_{C}^{(0)}$ and $\boldsymbol{K}_{F F}^{(0)}$ is inherited from the integrands. Note that the constants $k_{C}, K_{C}, k_{T}$ and $k_{F}$ are all negative values, and $k_{D}, K_{T}$ and $K_{F}$ can be both positive and negative depending on the helicity of the flagellum. Detailed expressions for a helical flagellum are listed in appendix A.

The dynamics of the model bacteria possess six degrees of freedom associated with the rigid motion and two degrees of freedom associated with the angles, $\alpha$ and $\beta$, which designate the orientation of the rod flagellum (figure 3). With a constant 
separation from the wall boundary, we have in total eight degrees of freedom, and we will hereafter derive a linearized equation around the vertical spinning motion for the stability analysis.

We first decompose $\boldsymbol{\omega}=\boldsymbol{F} \cdot \boldsymbol{\omega}^{(0)}$ into tangential and normal components with respect to the flagellar axis $\boldsymbol{e}_{z}^{\prime \prime}$ as $\boldsymbol{B} \cdot \boldsymbol{\omega}=\boldsymbol{\omega}_{t}+\boldsymbol{\omega}_{n}$, where $\boldsymbol{\omega}_{t} \cdot \boldsymbol{e}_{z}^{\prime \prime}=\omega_{0}$ and $\boldsymbol{\omega}_{n} \cdot \boldsymbol{e}_{z}^{\prime \prime}=0$. With $\boldsymbol{R}=$ $\boldsymbol{B} \cdot \boldsymbol{F}$, we introduce the flagellar rotation velocities in the flagellum-fixed coordinates, $\boldsymbol{\omega}_{t}^{(0)}$ and $\boldsymbol{\omega}_{n}^{(0)}$, as $\boldsymbol{\omega}_{t}=\boldsymbol{R} \cdot \boldsymbol{\omega}_{t}^{(0)}$ and $\boldsymbol{\omega}_{n}=\boldsymbol{R} \cdot \boldsymbol{\omega}_{n}^{(0)}$. Similarly, we can write the bending and motor torque expressions by the use of those in the flagellum-fixed coordinates as $\boldsymbol{M}_{\text {elast }}=\boldsymbol{R} \cdot \boldsymbol{M}_{\text {elast }}^{(0)}$ and $\boldsymbol{M}_{\text {motor }}=\boldsymbol{R} \cdot \boldsymbol{M}_{\text {motor }}^{(0)}$.

Substituting these into (3.11), we obtain the effective cell-body dynamics,

$$
\left(\begin{array}{lll}
\boldsymbol{K}_{T T} & \boldsymbol{K}_{T R} & \boldsymbol{K}_{T F} \\
\boldsymbol{K}_{R T} & \boldsymbol{K}_{R R} & \boldsymbol{K}_{R F}
\end{array}\right)\left(\begin{array}{c}
\boldsymbol{U} \\
\boldsymbol{\Omega} \\
\boldsymbol{\omega}_{n}
\end{array}\right)=\left(\begin{array}{l}
-\boldsymbol{K}_{T F} \cdot \boldsymbol{\omega}_{t} \\
-\boldsymbol{K}_{R F} \cdot \boldsymbol{\omega}_{t}
\end{array}\right),
$$

where the right-hand side vector represents the force and torque generated by the flagellar propulsion. The equations of motion for the flagellar bending are calculated as

$$
\begin{gathered}
\boldsymbol{P}_{0} \cdot \boldsymbol{K}_{F T}^{(0)} \cdot \boldsymbol{R}^{-1} \cdot \boldsymbol{U}+\boldsymbol{P}_{0} \cdot \boldsymbol{R}^{-1} \cdot \boldsymbol{K}_{F R} \cdot \boldsymbol{\Omega}+\boldsymbol{K}_{F F}^{(0)} \cdot \boldsymbol{\omega}_{n}^{(0)}=-\boldsymbol{M}_{\text {elast }}^{(0)}-\boldsymbol{P}_{0} \cdot \boldsymbol{M}_{\text {motor }}^{(0)}, \\
\boldsymbol{Q}_{0} \cdot \boldsymbol{K}_{F T}^{(0)} \cdot \boldsymbol{R}^{-1} \cdot \boldsymbol{U}+\boldsymbol{Q}_{0} \cdot \boldsymbol{R}^{-1} \cdot \boldsymbol{K}_{F R} \cdot \boldsymbol{\Omega}+\boldsymbol{K}_{F F}^{(0)} \cdot \boldsymbol{\omega}_{t}^{(0)}=-\boldsymbol{Q}_{0} \cdot \boldsymbol{M}_{\text {motor }}^{(0)}
\end{gathered}
$$

after projection into the tangential (3.16) and normal (3.17) components with respect to $\boldsymbol{e}_{z}^{\prime \prime}$, where $\boldsymbol{Q}_{0}=\boldsymbol{e}_{z} \boldsymbol{e}_{z}$ and $\boldsymbol{P}_{0}=\mathbf{1}-\boldsymbol{e}_{z} \boldsymbol{e}_{z}$ are the projections onto the $\boldsymbol{e}_{z}$ axis and the $x-y$ plane.

Let $\boldsymbol{R}_{0}(\phi, \boldsymbol{e})$ be a rotation matrix of degree $\phi$ around the vector $\boldsymbol{e}$; then the matrix $\boldsymbol{F}$ can be expressed by $\boldsymbol{F} \simeq \boldsymbol{R}_{0}\left(-\beta, \boldsymbol{e}_{x}\right) \cdot \boldsymbol{R}_{0}\left(\alpha, \boldsymbol{e}_{y}\right)$ for small angles, $\alpha$ and $\beta$. The local angular velocity can be obtained from the equation $\dot{\boldsymbol{F}}=\boldsymbol{\omega} \times \boldsymbol{F}$, and we find $\boldsymbol{\omega}_{n}^{(0)} \simeq-\dot{\beta} \boldsymbol{e}_{x}+\dot{\alpha} \boldsymbol{e}_{y}$. Similarly, $\boldsymbol{R}=\boldsymbol{B} \cdot \boldsymbol{F}$ are calculated as

$$
\boldsymbol{R}=\left(\begin{array}{ccc}
1 & 0 & \theta+\alpha \\
0 & 1 & \beta \\
-(\theta+\alpha) & -\beta & 1
\end{array}\right)+\text { higher-order terms }
$$

Using these expressions, we estimate the leading-order contributions of (3.17), which reads

$$
K_{F}\left(\Omega_{z}+\omega_{0}\right)=-\tau,
$$

noting that we fix the boundary separation distance and thus $U_{z}=0$. Similarly, from the last row of (3.15), we obtain the leading-order contributions

$$
\left(C_{R_{z}}+K_{F}\right) \Omega_{z}=-K_{F} \omega_{0} .
$$

Equations (3.19) and (3.20) provide the steady state that corresponds to the upright spinning motion, and we will consider the linearized equation around this equilibrium.

We begin by calculating the matrix entries in (3.15). By neglecting quadratic values of angles, we have

$$
\boldsymbol{K}_{T T}=\left(\begin{array}{ccc}
C_{D} & 0 & 0 \\
0 & C_{D} & 0 \\
0 & 0 & C_{D_{Z}}
\end{array}\right)+\boldsymbol{R} \cdot \boldsymbol{K}_{C}^{(0)} \cdot \boldsymbol{R}^{-1}
$$




$$
\simeq\left(\begin{array}{ccc}
C_{D}+k_{C} & 0 & \left(K_{C}-k_{C}\right)(\theta+\alpha) \\
0 & C_{D}+k_{C} & \left(K_{C}-k_{C}\right) \beta \\
\left(K_{C}-k_{C}\right)(\theta+\alpha) & \left(K_{C}-k_{C}\right) \beta & C_{D_{Z}}+K_{C}
\end{array}\right),
$$

and, introducing the matrix $\boldsymbol{A}^{\prime}=\boldsymbol{A}-\tilde{\boldsymbol{A}}$, we calculate

$$
\begin{aligned}
\boldsymbol{K}_{T R} & =\left(\begin{array}{ccc}
0 & -C_{S} & 0 \\
C_{S} & 0 & 0 \\
0 & 0 & 0
\end{array}\right)+\boldsymbol{K}_{C} \cdot \boldsymbol{A}^{\prime}+\boldsymbol{K}_{T F} \\
& \simeq\left(\begin{array}{ccc}
k_{D} & -C_{S}+\left(k_{C}+k_{T}\right) & -k_{T} \beta+K_{T}(\theta+\alpha) \\
C_{S}-\left(k_{C}+k_{T}\right) & k_{D} & k_{C} \theta+k_{T}(\theta+\alpha)+K_{T} \beta \\
* & * & K_{T}
\end{array}\right) .
\end{aligned}
$$

Here, the entries indicated by $*$ are of the order of $O(|\theta|,|\alpha|,|\beta|)$ and therefore provide higher-order contributions to the $z$ component of the torque balance equation. We also obtain

$$
\begin{aligned}
\boldsymbol{K}_{R T} & =\left(\begin{array}{ccc}
0 & -C_{S}^{\prime} & 0 \\
C_{S}^{\prime} & 0 & 0 \\
0 & 0 & 0
\end{array}\right)+\left[\boldsymbol{K}_{C} \cdot \boldsymbol{A}^{\prime}+\boldsymbol{K}_{T F}\right]^{\mathrm{T}} \\
& \simeq\left(\begin{array}{ccc}
k_{D}^{\prime}-\left(k_{C}+k_{T}\right) & -C_{S}^{\prime}+\left(k_{C}+k_{T}\right) & * \\
* & k_{D} & * \\
& * & K_{T}
\end{array}\right),
\end{aligned}
$$

and the entries shown by $*$ are the higher-order contributions that do not appear in the linearized equations. Combining the above expressions, we finally have

$$
\begin{aligned}
\boldsymbol{K}_{R R} & =\left(\begin{array}{ccc}
C_{R} & 0 & 0 \\
0 & C_{R} & 0 \\
0 & 0 & C_{R_{Z}}
\end{array}\right)+\boldsymbol{A}^{\prime \mathrm{T}} \cdot \boldsymbol{K}_{C} \cdot \boldsymbol{A}^{\prime}+\boldsymbol{A}^{\prime \mathrm{T}} \cdot \boldsymbol{K}_{F T}+\boldsymbol{K}_{F T} \cdot \boldsymbol{A}^{\prime}+\boldsymbol{K}_{F F} \\
& \simeq\left(\begin{array}{ccc}
\bar{C}_{R} & 0 & K_{R_{x}} \\
0 & \bar{C}_{R} & K_{R_{y}} \\
K_{R_{x}} & K_{R_{y}} & C_{R_{z}}+K_{F}
\end{array}\right)
\end{aligned}
$$

where $\bar{C}_{R}=C_{R}+k_{C}+2 k_{T}+k_{F}, K_{R_{x}}=-\left(k_{C}+k_{T}\right) \theta+\left(K_{F}-k_{F}-k_{T}\right)(\theta+\alpha)-K_{T} \beta$ and $K_{R_{y}}=K_{T} \alpha+\left(K_{F}-k_{F}-k_{T}\right) \beta$.

The right-hand side of (3.15) is also calculated using $\omega^{(0)}=\omega_{0} \boldsymbol{e}_{z}$ as

$$
\begin{gathered}
\boldsymbol{K}_{T F} \cdot \boldsymbol{\omega}_{t}=\boldsymbol{R} \cdot \boldsymbol{K}_{T F}^{(0)} \cdot\left(\omega_{0} \boldsymbol{e}\right)_{z}=\omega_{0} K_{T}\left\{(\theta+\alpha) \boldsymbol{e}_{x}+\beta \boldsymbol{e}_{y}\right\}, \\
\boldsymbol{K}_{R F} \cdot \boldsymbol{\omega}_{t}=\omega_{0} K_{F}\left\{(\theta+\alpha) \boldsymbol{e}_{x}+\beta \boldsymbol{e}_{y}+\boldsymbol{e}_{z}\right\}+\omega_{0} K_{T}\left(-\beta \boldsymbol{e}_{x}+\alpha \boldsymbol{e}_{y}\right) .
\end{gathered}
$$

We substitute these relations into (3.16) and (3.17), and finally obtain

$$
\left.\begin{array}{c}
-k_{D} U_{x}+k_{T} U_{y}-\left(k_{T}+k_{F}\right) \Omega_{x}-k_{D} \Omega_{y}+\left(\left(k_{T}+k_{F}\right) \theta+k_{F} \alpha\right) \Omega_{z}+k_{F} \dot{\beta}=\kappa \beta-M_{0} \alpha, \\
k_{T} U_{x}+k_{D} U_{y}-k_{D} \Omega_{x}+\left(k_{T}+k_{F}\right) \Omega_{y}-k_{F} \beta \Omega_{z}+k_{F} \dot{\alpha}=\kappa \alpha+M_{0} \beta .
\end{array}\right\}
$$

Noting that $\Omega_{y}=\dot{\theta}$ and introducing $\dot{\boldsymbol{X}}=\left(U_{x}, \dot{\theta}, \dot{\alpha}, U_{y}, \Omega_{x}, \dot{\beta}\right)^{\mathrm{T}}$, these equations (3.21)-(3.26) can be summarized into the following matrix form:

$$
\mathcal{A} \dot{\boldsymbol{X}}=\mathcal{B} \boldsymbol{X},
$$


with

$$
\mathcal{A}=\left(\begin{array}{ll}
\boldsymbol{A}_{1} & \boldsymbol{A}_{3} \\
\boldsymbol{A}_{3}^{\mathrm{T}} & \boldsymbol{A}_{2}
\end{array}\right),
$$

where $\boldsymbol{A}_{1}, \boldsymbol{A}_{2}$ and $\boldsymbol{A}_{1}$ are $3 \times 3$ matrices, given by

$$
\begin{gathered}
\boldsymbol{A}_{1}=\left(\begin{array}{ccc}
C_{D}+k_{C} & -C_{S}+\left(k_{C}+k_{T}\right) & k_{T} \\
-C_{S}^{\prime}+\left(k_{C}+k_{T}\right) & C_{R}+k_{C}+2 k_{T}+k_{F} & k_{T}+k_{F} \\
k_{T} & k_{T}+k_{F} & k_{F}
\end{array}\right), \\
\boldsymbol{A}_{2}=\left(\begin{array}{ccc}
C_{D}+k_{C} & C_{S}^{\prime}-\left(k_{C}+k_{T}\right) & k_{T} \\
C_{S}^{\prime}-\left(k_{C}+k_{T}\right) & C_{R}+k_{C}+2 k_{T}+k_{F} & k_{T}+k_{F} \\
k_{T} & -\left(k_{T}+k_{F}\right) & k_{F}
\end{array}\right), \\
\boldsymbol{A}_{3}=\left(\begin{array}{ccc}
0 & k_{D} & -k_{D} \\
k_{D} & 0 & -k_{D} \\
k_{D} & -k_{D} & 0
\end{array}\right),
\end{gathered}
$$

and $\mathcal{B}$ is a $6 \times 6$ matrix whose components are

$$
\mathcal{B}=\left(\begin{array}{cccccc}
0 & -F_{e} & -F_{e} & 0 & 0 & k_{T} \Omega_{z} \\
0 & 0 & -F_{e} & 0 & 0 & \left(k_{T}+k_{F}\right) \Omega_{z}-M_{e} \\
0 & 0 & \kappa & 0 & 0 & k_{F} \Omega_{z}+\tau \\
0 & -\left(k_{C}+k_{T}\right) \Omega_{z} & -k_{T} \Omega_{z} & 0 & 0 & -F_{e} \\
0 & \left(k_{C}+2 k_{T}+k_{F}\right) \Omega_{z}-M_{e} & \left(k_{T}+k_{F}\right) \Omega_{z}-M_{e} & 0 & 0 & F_{e} \\
0 & -\left(k_{T}+k_{F}\right) \Omega_{z} & -k_{F} \Omega_{z}-\tau & 0 & 0 & \kappa
\end{array}\right) .
$$

In the expression (3.33), we have introduced the effective force and torque associated with the flagellar rotation by $F_{e}=K_{T}\left(\Omega_{z}+\omega_{0}\right)$ and $M_{e}=K_{F}\left(\Omega_{z}+\omega_{0}\right)$, respectively.

From (3.33), it is readily found that the cell rigid motion obeys the angle dynamics, which can be obtained by inverting the matrix $\mathcal{A}$ as

$$
\frac{\mathrm{d}}{\mathrm{d} t}\left(\begin{array}{c}
\theta \\
\alpha \\
\beta
\end{array}\right)=\boldsymbol{A}_{L}\left(\begin{array}{l}
\theta \\
\alpha \\
\beta
\end{array}\right),
$$

and the eigenvalues of the matrix $\boldsymbol{A}_{L}$ provide the linear stability of the upright spinning motion.

In figure 4 , the largest real parts of the eigenvalues are plotted for different values of the cell-body size $L_{e} / a$ and dimensionless hook rigidity $\kappa / \tau$. The effective length of the axisymmetric flagellum is $L_{e} \approx 0.85 \mathrm{~L}$ for the parameters of the model $P$. aeruginosa (flagellum 1 in table 1 ). The separation distance from the boundary is $h / L_{e}=0.01$ for figure $4(a, c)$ and $h / L_{e}=0.001$ for figure $4(b, d)$. When $\tau>0$, the flagellum generates a force towards the cell body, as of pushers, and the results are shown in figure $4(a, b)$. With a negative $\tau$, the rotation direction of the flagellum is inverted, and the propulsive force pulls away from the cell body, as of pullers, and figure $4(c, d)$ correspond to this case.

As seen in figure 4, the linear stability dramatically differs between the pusher and puller flagella, and the pusher flagellum can be stable only when the dimensionless rigidity ranges in an intermediate magnitude, $\kappa / \tau \sim 1$. In contrast, the puller flagellum 


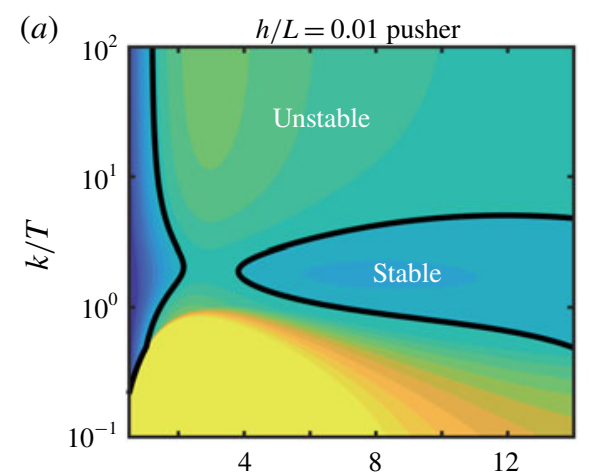

(b)
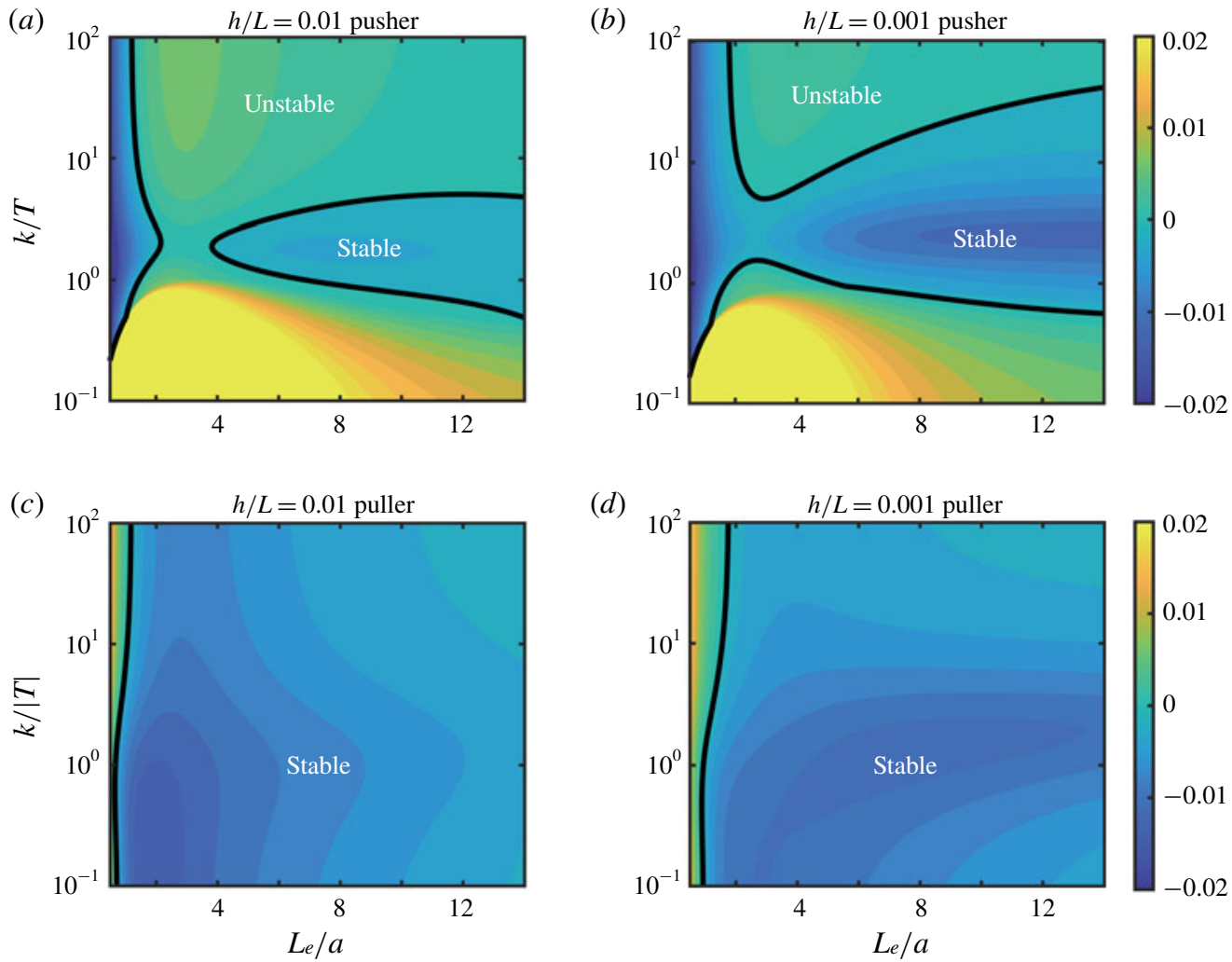

FIgURE 4. (Colour online) The largest real parts of the eigenvalues in the linear stability analysis for different cell-body size and hook rigidity. (a) The separation distance is $h / L_{e}=0.01$ and the flagellum is rotated by motor torque to push forwards to the cell body (pusher). (b) The separation distance is $h / L_{e}=0.001$ and the flagellum rotation direction is the same (pusher) as in $(a)$. (c) The flagellar rotation direction is inverted (puller) from $(a, b)$ with the separation distance, $h / L_{e}=0.01$. (d) The separation distance is $h / L_{e}=0.001$ and the flagellar rotation direction is the same (puller) as in (c).

case is found to be stable in a broad range of the cell-body size and hook rigidity. The stability phase map, however, is not rigorously symmetric, reflecting the breakdown of the pusher-puller duality by the flexibility of the hook. With a completely rigid flagellum, the swimming stability is shown to be opposite between the pusher and the corresponding puller from the time-reversal symmetry of the Stokes equations (Ishimoto \& Gaffney 2013). The effects of the separation distance are more significant in the pusher cases, and the parameter regions for stable spinning motions are broadened as the cell body approaches the boundary.

The linearized problem (3.34) contains both the force and torque contributions of the flagellar propulsion. To examine the mechanisms underlying the non-trivial stability phase map, we will theoretically separate the force and torque contributions.

We first consider the contribution from the flagellar force exertion, dropping the flagellar torque effects by setting $\Omega_{z}=0$ and $M_{e}=0$ while keeping $F_{e}$ being non-zero in the matrix $\mathcal{B}$. The sign of $\tau$ determines whether the flagellum is a pusher or a puller. We calculate the largest real parts of the eigenvalues in the linear problem without torque terms, and the results are plotted in figure $5(a, b)$ for the case of $h / L_{e}=0.01$, 

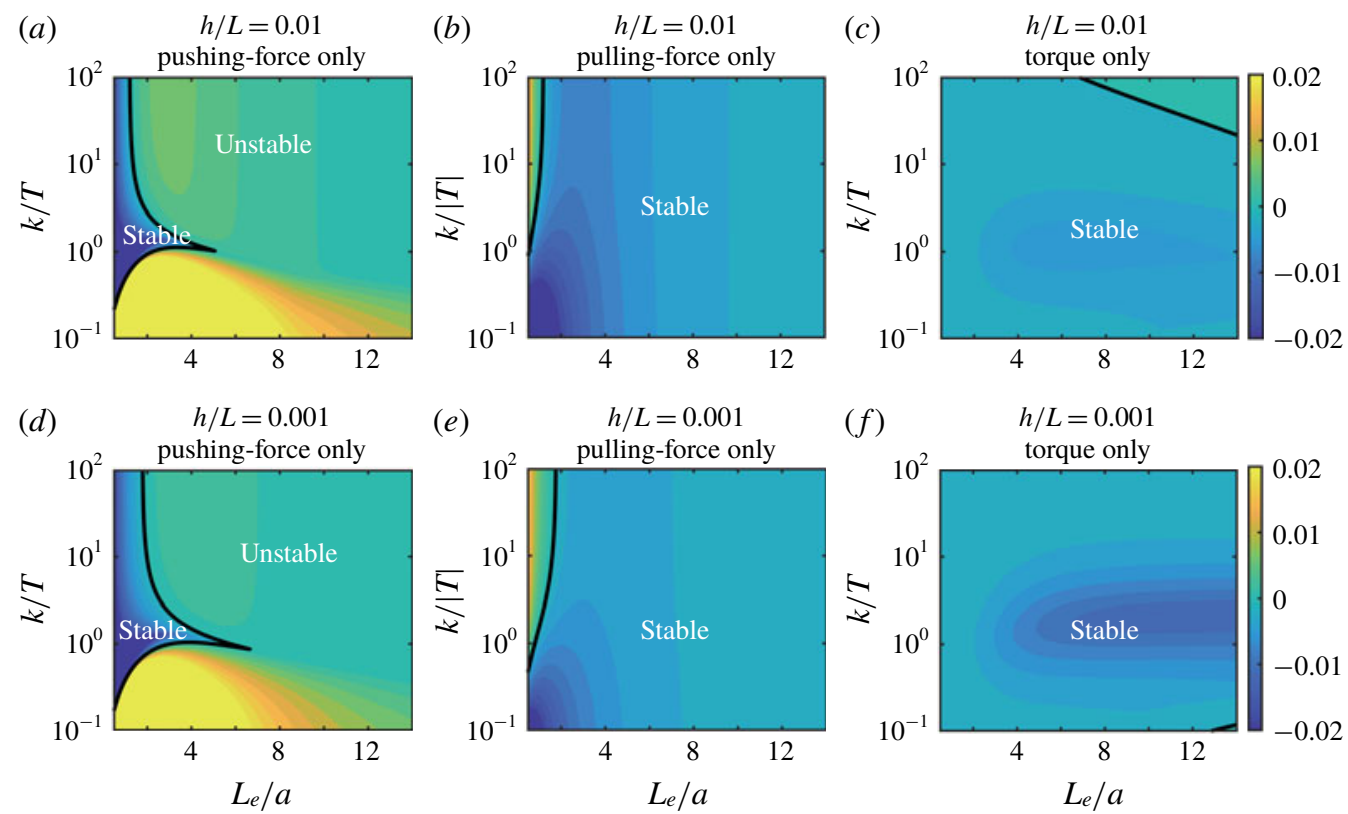

FIgURE 5. (Colour online) The largest real parts of the eigenvalues in the linear stability analysis neglecting either the force or torque contribution from the flagellar propulsion. The separation distance is $h / L_{e}=0.01(a-c)$ and $h / L_{e}=0.001(d-f)$. Panels $(a, d)$ and $(b, e)$ show the linear stability for the flagellum without torque exertion (pushing force only, pulling force only), and panels $(c, f)$ correspond to the results for the flagellum without force exertion (torque only).

and in figure $5(d, e)$ when $h / L_{e}=0.001$. As shown in figure $5(a, d)$ (pushing force only), the stability of a pushing flagellar bacterium is not as simple as previously considered (Lauga et al. 2006; Petroff \& Libchaber 2018), whereas a puller flagellum stabilizes the upright position in a wider range of parameters (figure $5 b, e$, pulling force only).

To provide physical interpretations for the problem without the torque effects, we again consider the full problem of (3.34), where the expression of the matrix $\boldsymbol{A}_{L}$ requires the inverse of the $6 \times 6$ matrix $\mathcal{A}$. When the flagellar chirality effects $k_{D}$ can be negligible, the matrix inverse can be simplified, since the off-diagonal block $\boldsymbol{A}_{3}$ vanishes. In the realistic bacterial flagellar shape, this term is typically very small, with the relative magnitude of the order of $O\left(10^{-2}\right)$ compared with the other terms (Ishimoto \& Lauga 2019). We therefore temporarily neglect the chirality effects by $k_{D}=0$ and consider the mathematical structures of the linear equation (3.34). Even with this simplification, the dynamics are still complicated since the angle $\beta$ dynamics is strongly coupled with the entire motion as seen in the right-most column of (3.33). With further simplification by assuming that the angle $\beta$ is decoupled from the entire dynamics, considering the hook being allowed to bend in one direction, we can reduce (3.34) to the form

$$
\frac{\mathrm{d}}{\mathrm{d} t}\left(\begin{array}{c}
\theta \\
\alpha
\end{array}\right)=\boldsymbol{A}_{0}\left(\begin{array}{c}
\theta \\
\alpha
\end{array}\right),
$$

which coincides with the dynamics without the flagellar torque effects under the assumption of negligible chirality effects, and therefore the maximum eigenvalue 
of the matrix $\boldsymbol{A}_{0}$ is very close to the plots shown in figure $5(a, b, d, e)$. The force contribution can therefore be interpreted as the dynamics without the dynamics associated with the angle $\beta$.

As in figure $5(a, d)$, except for the region with $\kappa / \tau \sim 1$ in the pusher flagellum case, the stability is mainly determined by the flagellar length $L_{e} / a$, and this can be simply understood by analysing the rigid hook limit $(\kappa \rightarrow \infty)$. Let us again go back to the full problem (3.34) and neglect the small chirality effects for brevity. We can dismiss the dynamics of the angles $\alpha$ and $\beta$ at the rigid hook limit, and the stability can be obtained by the two-dimensional linear problem,

$$
\left(\begin{array}{cc}
C_{D}+k_{C} & -C_{S}+\left(k_{C}+k_{T}\right) \\
-C_{S}^{\prime}+\left(k_{C}+k_{T}\right) & C_{R}+k_{C}+2 k_{T}+k_{F}
\end{array}\right)\left(\begin{array}{c}
U_{x} \\
\dot{\theta}
\end{array}\right)=\left(\begin{array}{c}
F_{e} \theta \\
0
\end{array}\right) .
$$

Inverting the matrix on the left-hand side, we obtain the time evolution of angle $\theta$,

$$
\frac{\mathrm{d} \theta}{\mathrm{d} t}=-\frac{F_{e}}{\Delta_{r}}\left[C_{S}^{\prime}-\left(k_{C}+k_{T}\right)\right] \theta,
$$

where $\Delta_{r}$ is the determinant of the $2 \times 2$ matrix of (3.36) and is a positive value.

The longer flagellum experiences a large hydrodynamic drag that orients the cell towards the wall boundary as $k_{C}=O\left(L_{e}\right)$ and $k_{T}=O\left(L_{e}^{2}\right)$, whereas $C_{S}^{\prime}$ is independent of $L_{e}$ and behaves as $O\left(a^{3} \log (a / h)\right)$ from the lubrication theory (see also appendix A). We therefore obtain a critical flagellar length $L_{e}^{*}$ as a function of separation distance $h$, above which the vertical spinning configuration becomes unstable for the pusher flagellum case. From the time-reversal symmetry of the Stokes equations, the critical value provides the lower limit of the flagellar length for a stable spinning top with a puller flagellum at the rigid limit. Further, these results indicate that the rigid hook model cannot exhibit a stable spinning top motion if the flagellum length is reasonably long as in a real bacterial swimmer $\left(L_{e} / a \sim 10\right)$.

We then consider the contribution from the flagellar torque exertion. We drop the flagellar force effects simply by setting $F_{e}=0$ and keep $\Omega_{z}$ and $M_{e}$ being non-zero in the matrix $\mathcal{B}$. As in the flagellar force contribution, we compute the largest real parts of the eigenvalues of the linear problem with the flagellar force terms. The results are plotted in figure $5(c)$ for the case of $h / L_{e}=0.01$ and figure $5(f)$ for the case of $h / L_{e}=0.001$. Note that the stability is independent of the sign of $\tau$ due to the symmetry.

From these plots (figure $5 c, f$ ), it is found that the torque due to the flagellar propulsion contributes to the stabilization of the upright spinning motion in a large parameter region, and, in particular, with the intermediate hook rigidity, $\kappa / \tau \sim 1$, the dynamical stabilization is promoted for a relatively smaller cell body (or a longer flagellum), including the biologically relevant parameter regimes $L_{e} / a \sim 10$. The hook rigidity of $P$. aeruginosa has been estimated as $\kappa / \tau \approx 2$ (de Anda et al. 2017), which remarkably lies in the region where the vertical spinning motion is strongly stabilized.

Figure $6(a, b)$ indicates the summations of the largest real parts of the eigenvalues from the force-only and torque-only problems in figure 5. In figure $6(a)$, the sum of the values of figures 5(a) and 5(c) are shown. This stability map for the pusher flagellum with $h / L_{e}=0.01$ is almost the same as the full linear problem in figure $4(a)$. Similarly, the sum of the values of figures $5(d)$ and $5(f)$ are shown in figure $6(a)$ for the pusher flagellum with $h / L_{e}=0.001$. We find a remarkable coincidence on the stability map between the full problem and the sum of the force-only and torque-only 

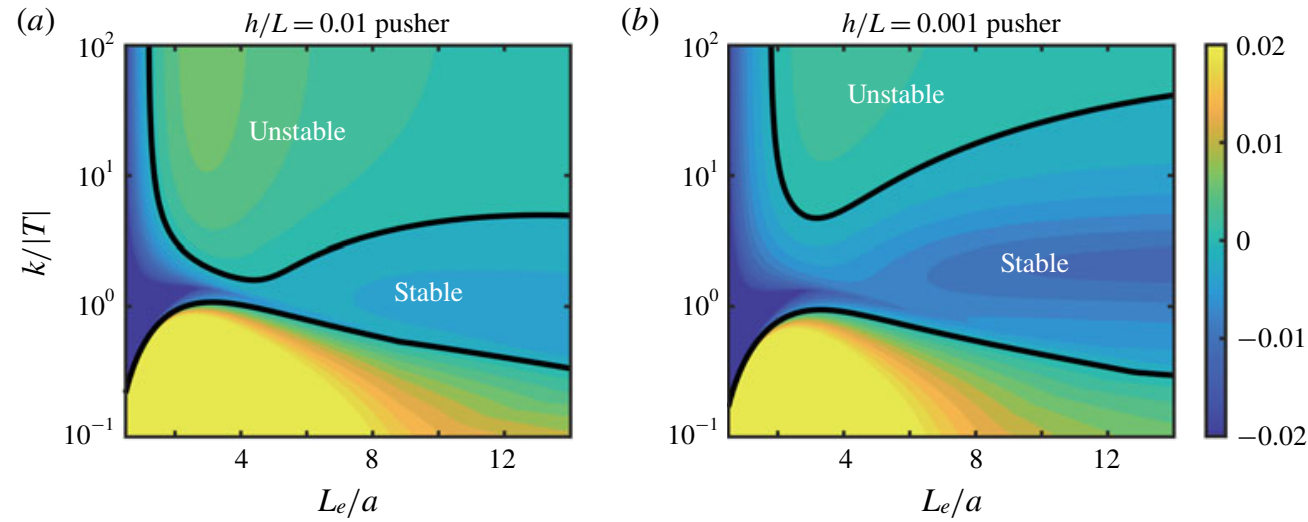

FIgURE 6. (Colour online) The summation of the largest real parts of the eigenvalues from the force-only and torque-only problems for a pusher flagellum. (a) Plots obtained by the summation of the values in figure $5(a, c)$, where the separation distance is $h / L_{e}=0.01$. (b) Plots obtained by the summation of the values in figure $5(d, f)$, where the separation distance is $h / L_{e}=0.001$.

problems. We can thus understand the full stability analysis in figure 4 by the separate effects of the flagellar force and torque contributions shown in figure 5.

These enable us to simply interpret the mechanical stability of the vertical spinning motion as the competition between the flagellar force and torque contributions. With the counter-rotating pulling flagellum, the flagellar propulsive force contributes to the stable vertical motion, whereas the propulsive force contributes to destabilizing the spinning motion in the pusher flagellar case. In a limited but biologically relevant parameter region, however, the torque stabilization effects are dominating compared with the force destabilization effect, hence the vertical spinning results in a mechanically stable state.

\section{Simulation results I: constant wall separation}

In the following two sections, we will present the simulation results of bacterial motility, and we start with the case of a constant separation distance $h$.

\subsection{Spherical cell body}

The linear stability analysis based on the local interaction theory in the previous section has suggested that the spherical cell body with a pusher flagellum can be stabilized only when the magnitudes of the flagellar bending torque and induced motor torque are comparable, i.e. $\kappa / \tau \sim 1$. We will see how the full hydrodynamic interactions could be explained by the local interaction theory.

Following figure 4 , the swimming behaviours have been numerically examined for different cell body/flagellar length ratio $a / L$ and hook rigidity $\kappa / \tau$. At each parameter value, we have performed numerical simulations with two different initial conditions of the shallow initial angle $\theta_{\text {init }}=0.1 \pi$ and steeper angle $\theta_{\text {init }}=0.25 \pi$. Throughout this paper, the initial position of the cell-body centre satisfies $x / L=y / L=0$, and the initial bending angle is set to be zero, i.e. $\varphi_{\text {init }}=0$.

The stability diagrams are summarized in figure 7 for the different separation distances, $h / L=0.01$ and 0.001 . Depending on the long-time behaviours obtained 


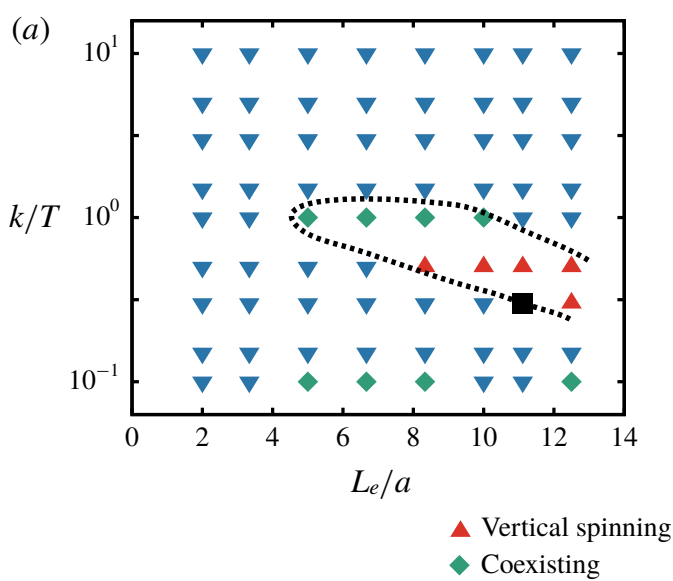

(b)

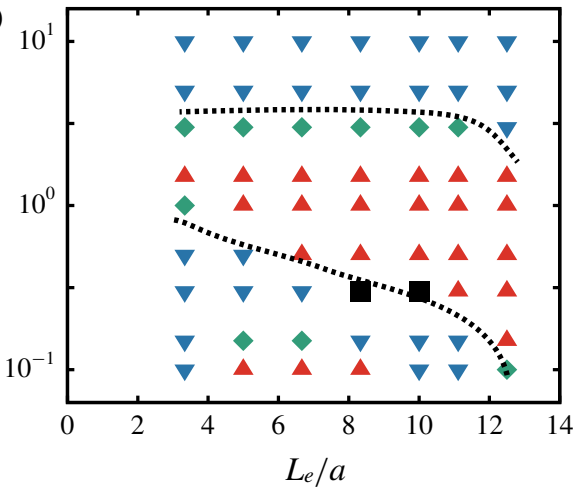

Non-vertical spinning

$\nabla$ Boundary following

FIgURE 7. (Colour online) Swimming behaviours of a model bacterium with a spherical cell body and a pusher flagellum with constant separation distances of $(a) h / L=0.01$ and $(b) h / L=0.001$. For each parameter value, the simulations have been done with two different initial angles, $\theta_{\text {init }}=0.1 \pi$ and $\theta_{\text {init }}=0.25 \pi$. We enclose by dashed lines the region with stable upright spinning motions, which contains the cases when stable vertical motions are observed from both initial angle conditions (red upright triangle $\mathbf{\Delta}$ ) and only the shallow initial angle (green diamond $\diamond$ ). The upright spinning motion cannot be realized in the parameter regions indicated by blue down triangles $(\boldsymbol{\nabla})$. In the region indicated by black rectangles ( $\boldsymbol{\square})$, the inclination angle $\theta$ approaches a non-zero value, and the bacterium exhibits a non-vertical spinning motion.

from the numerical simulations, the dynamics are categorized into the four groups, which are plotted in different colours and symbols. The red upright triangles $(\mathbf{\Delta})$ indicate that the stable vertical spinning motion is realized from the two different initial angles. In the parameters indicated by the green diamonds $(\bullet)$, only the shallow initial angle case has exhibited the stable upright motion, and the steeper initial angle has resulted in a wall-following behaviour, whereas the cell has moved along the boundary for both the initial angles in the parameter regions indicated by the blue down triangles $(\boldsymbol{\nabla})$. The black squares ( $\boldsymbol{\square})$ represent the cases where the upright spinning motion is obtained but the inclination angle approaches a non-trivial angle.

The regions enclosed by the dashed lines in figure 7 contain the parameter sets for the stable upright spinning motion, which may correspond to the linear stable regions obtained in the local interaction theory. We found remarkable agreement between the local interaction theory and the full numerical simulation. In particular, the enhancement of the stability by the intermediate hook rigidity, $\kappa / \tau \sim 1$, has been successfully predicted by the linear stability theory. Nevertheless, the stable behaviours in the very flexible parameter regions are not explained by the local interaction theory. In these parameter regions, the flagellar bending angles are oscillatory in time (see also figures 8 and 9), and the axisymmetric flagellar propulsion model would not be a feasible assumption. Another subtle disagreement between the theory and simulation can be found in the enlargement of the stable region with large values of $L_{e} / a$. The eigenvalue plots in figure 4 indicate that vertical motion is weakly stable in this region, and thus the simulation may have missed the basin of the weak attractor.

In contrast to the rich behaviours of the pusher flagellar dynamics, the puller behaviour of the backward flagellar rotation is found to be much simpler. We found 

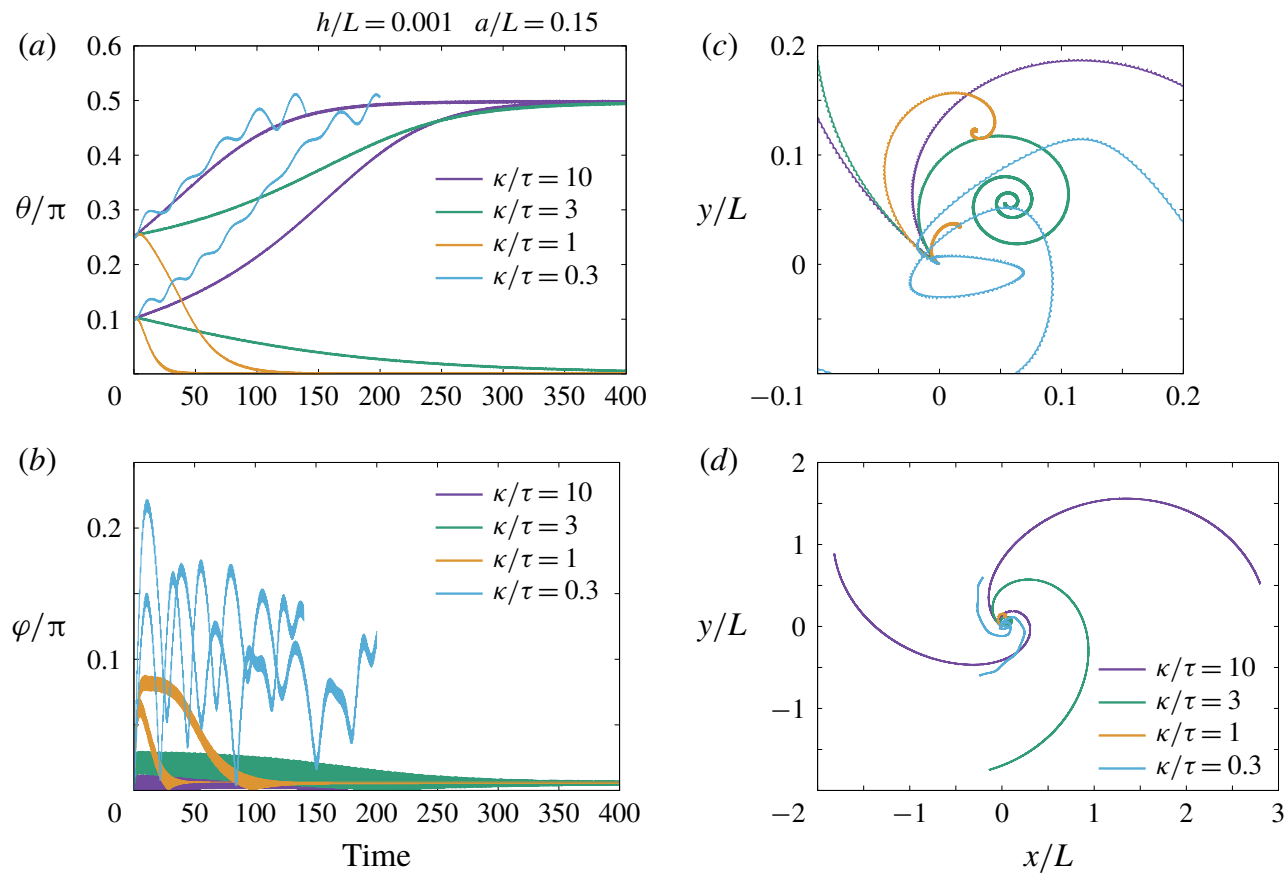

FIgURE 8. (Colour online) Dynamics of a model bacterial swimmer with a spherical cell body of radius $a / L=c / L=0.15$. The motor torque is given by a non-dimensional value of 0.04 , and the results of different hook rigidity $\kappa / \tau=\{10,3,1,0.3\}$ are shown. The separation distance is fixed as $h / L=0.001$. $(a, b)$ The time evolutions of the cell-body inclination angle $\theta(a)$ and the flagellar bending angle $\varphi(b)$ are plotted in units of $\pi$. $(c, d)$ The trajectories of the cell-body centre for the same simulations are shown in a closer view $(c)$ and in a wider view $(d)$. The simulation of $\kappa / \tau=0.3$ had to be terminated around the non-dimensional time of 100-200, as the flagellum approaches much too close to the boundary.

in all the simulations for a puller flagellum that stable upright spinning motion is exhibited, as we explored the same parameter region as in figure 7 (thus not shown as a figure). This again agrees with the prediction by the linear stability analysis of the local interaction theory.

To examine the robustness of these stability behaviours, we examined the different set of the parameters for the flagellum shape listed as flagellum 2 in table 1 . We have confirmed that the stability diagram for the new flagellar shape has been unchanged, with the same stability obtained for 48 different representative parameter values of $h / L, a / L, \kappa / \tau$ and $\theta_{\text {init }}$.

We then proceed to detailed dynamics of the bacterial motions. In figures 8 and 9 , the time evolutions of the angle variables, and the trajectories of the cell-body centre, are plotted for the simulations with the separation distance $h / L=0.001$ and the cellbody radius of $a / L=c / L=0.15$ (figure 8) and $a / L=c / L=0.1$ (figure 9).

In the case of the radius $a / L=c / L=0.15$, the inclination angle $\theta$ approaches $\theta \approx 0$ (upright spinning motion) or $\theta \approx \pi / 2$ (boundary-following motion), depending on the hook rigidity $\kappa / \tau$ and the initial angles (figure $8 a$ ). In contrast, the flagellar bending angle $\varphi$ finally reaches zero after a long time, irrespective of the final swimmer dynamics, except in the case of very flexible hook parameter $\kappa / \tau=0.3$ (figure $8 b$ ). The trajectory plots in figure $8(c, d)$ display the same data in different ranges, and we 

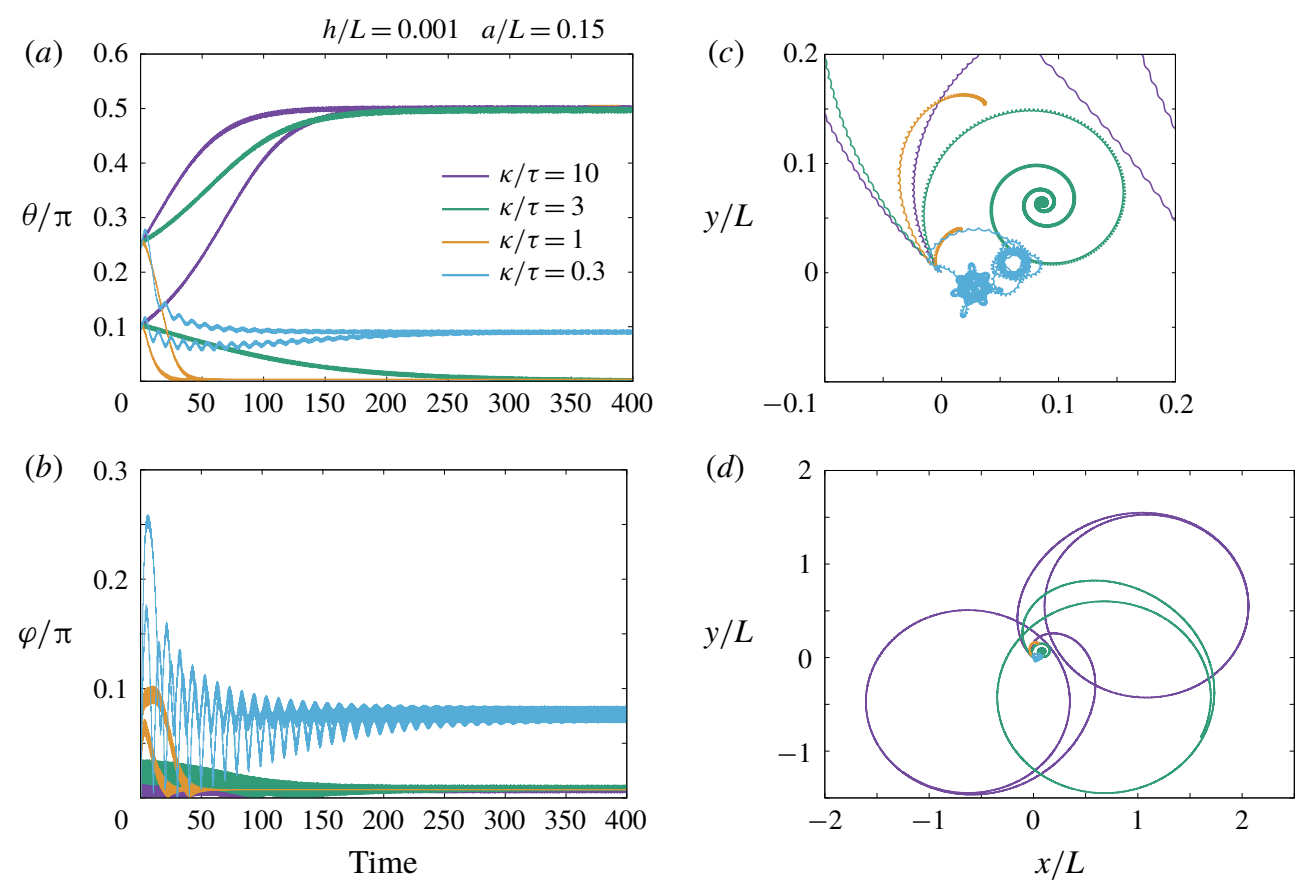

FIgURE 9. (Colour online) Dynamics of a model bacterial swimmer with a spherical cell body, as in figure 8 , but with a smaller radius $a / L=c / L=0.1$. The motor torque is given by a non-dimensional value of 0.04 , and the results of different hook rigidity $\kappa / \tau=$ $\{10,3,1,0.3\}$ are shown. $(a, b)$ The separation distance is fixed as $h / L=0.001$. The time evolutions of the cell-body inclination angle $\theta(a)$ and the flagellar bending angle $\varphi(b)$ are plotted in units of $\pi$. $(c, d)$ The trajectories of the cell-body centre for the same simulations are shown in a closer view $(c)$ and in a wider view $(d)$.

readily find that the cell ceases to move horizontally and starts to stay at a certain position with spinning vertically as the angle $\theta$ decreases to zero. When the hook rigidity is $\kappa / \tau=1$, the cell direction is rapidly oriented towards the vertical axis, while the bending angle $\varphi$ possesses a certain amount of non-zero values. These differences of the cell-body and flagellar orientation generate a torque that leads to the upright motion (de Anda et al. 2017), and the same mechanism can be found for the situation where the cell is allowed to move horizontally, being compatible with the theoretical prediction that the vertical spinning motion is stabilized by the coupling to the cell-body rotation. However, the simulation results of $\kappa / \tau=0.3$ show an oscillatory dynamics in $\varphi$, and the cell eventually swims along the boundary. Further, as the angle $\theta$ exceeds the value of $\theta=\pi / 2$, the flagellum finally approaches the boundary; thus the simulation has been terminated before the flagellum comes into the region very close to the boundary. To proceed with the simulation further, we would need additional assumptions on the flagellar-boundary interactions.

Similar plots are shown in figure 9 for the simulations with the cell-body radius $a / L=c / L=0.1$. As in figure 8 , we observe similar behaviour of the bacterial cell, and the misalignment between the cell and the flagellar orientations generates the torque that induces the upright spinning behaviour. But in the case with a flexible hook parameter, $\kappa / \tau=0.3$, the angles $\theta$ and $\varphi$ approach non-trivial values $\theta \approx \varphi \approx 0.1 \pi$, while the cell does not travel along the boundary but spins with its flagellar distal end being oriented almost towards the $z$ axis. 
(a)

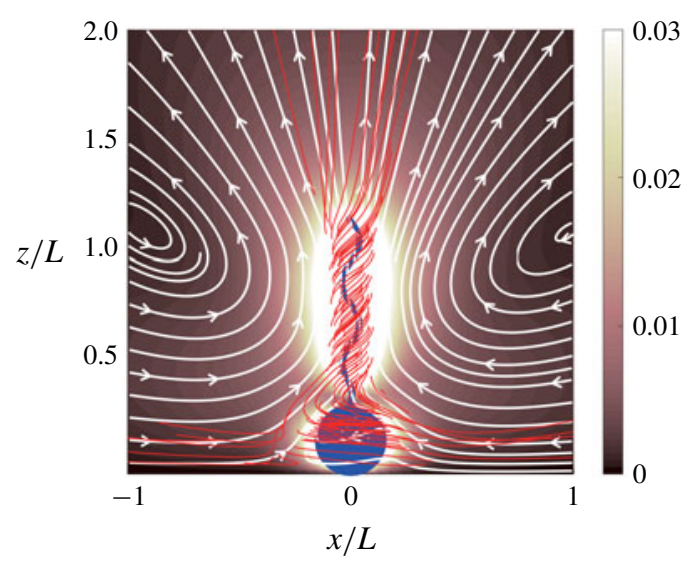

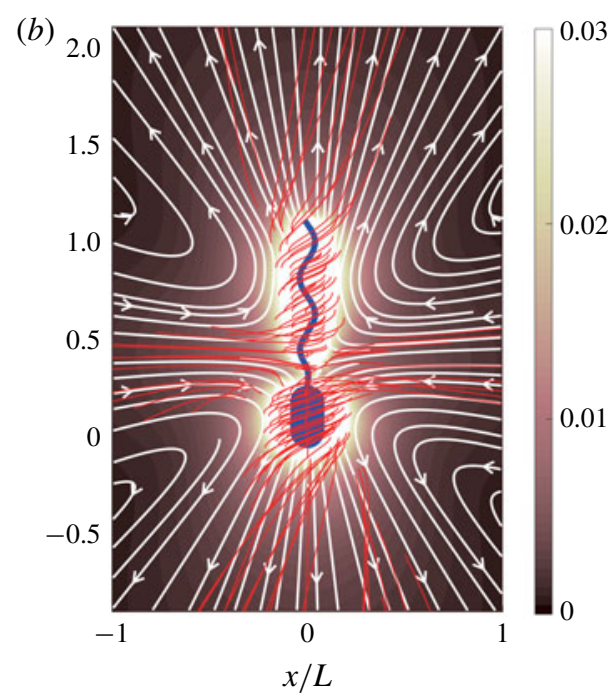

FIGURE 10. (Colour online) Time-averaged flow field around a swimming bacterium. (a) Time-averaged flow field around a cell with a spherical cell body of radius $a / L=c / L=$ 0.15 in a stable upright configuration near a wall boundary with a separation distance of $h / L=0.003$. (b) Time-averaged flow field around a cell with a spherocylindrical cell body of dimensions $(a / L, c / L)=(0.075,0.15)$ without external boundaries. Streamlines of the flow in the $x-z$ plane are shown by the white curves with arrows; the magnitudes of the velocity field are presented by the colour contour. The streamlines for the three-dimensional flow fields are projected onto the $x-z$ plane and shown by the red curves. The instantaneous bacterial shape is depicted in blue. The time average was taken with respect to the laboratory frame for the upright spinning cell $(a)$ and the body-fixed frame for the free-swimming cell $(b)$. The colour bar indicates the non-dimensional velocity, and the constant non-dimensional motor torque of 0.04 is used.

Before moving to the non-spherical cell-body results, we briefly discuss the flow field around the bacterial cell. With the parameters used in figure 8 , the intermediate size of the hook rigidity $\kappa / \tau=1$ exhibits the stable upright spinning motion. We have computed the flow field after the spinning motion is achieved, and its time average is shown in figure $10(a)$. The colour contour shows the magnitude of the velocity field, and the white lines with arrows indicate the streamline in the $x-z$ plane. The three-dimensional streamlines are shown in red lines, illustrating that the fluid near a boundary is attracted towards the cell body with swirling and then released from the distal end of the flagellum. The entire flow fields are well approximated by the rotating sphere near a wall combined with a flagellum represented by a rotlet and a stokeslet (Petroff et al. 2015). For comparison, the time-averaged flow field in free space is computed for the model bacterium with a spherocylindrical cell body (figure 10b); as is well known, the flow field is that around a pusher-type Stokes dipole (Drescher et al. 2011).

\subsection{Non-spherical cell body}

We have shown remarkable agreements between the theory and simulation for a spherical cell body; however, the actual bacterial cell usually possesses an elongated 

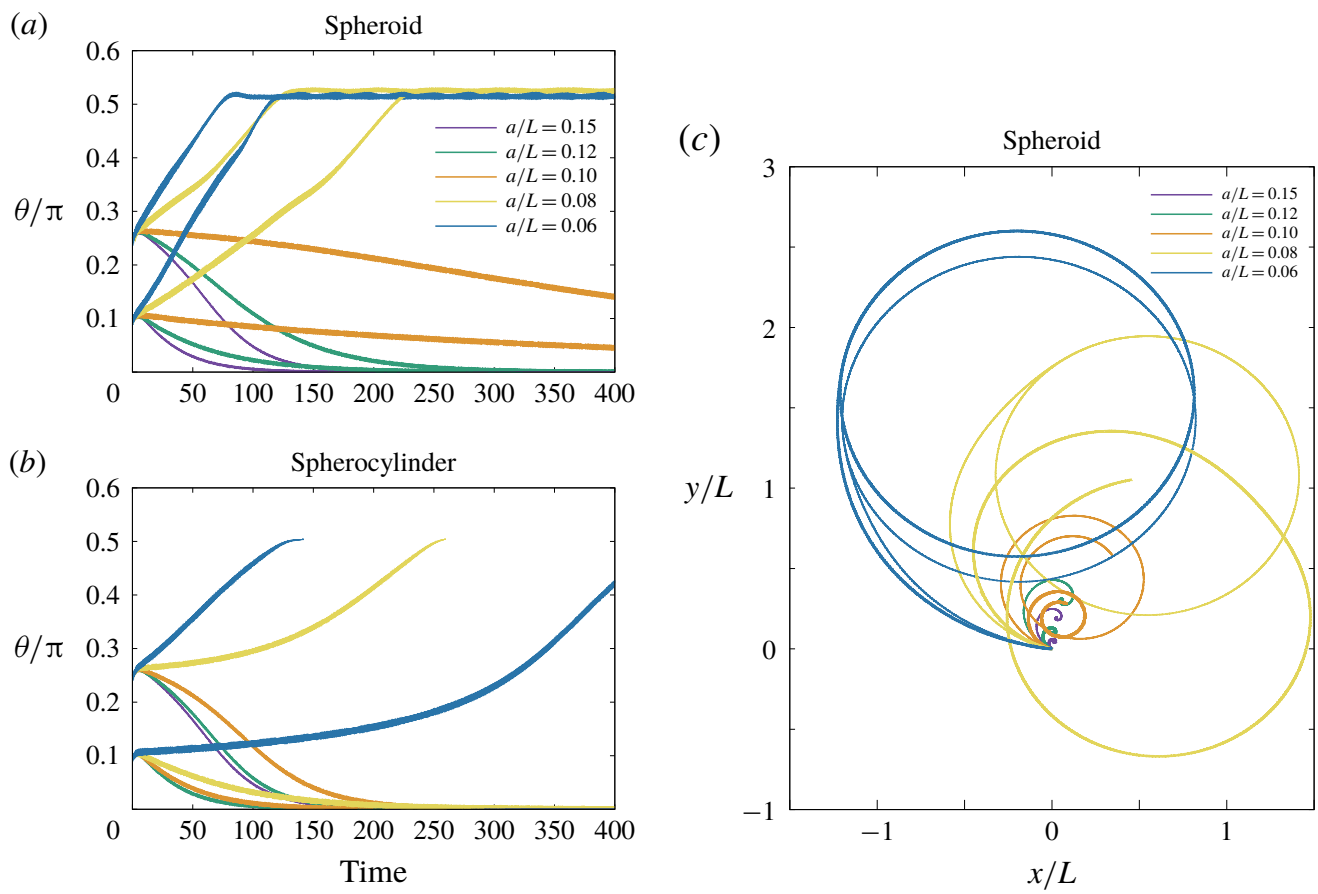

FIgURE 11. (Colour online) Dynamics of a model bacterial swimmer with a spheroidal and spherocylindrical cell body. The motor torque is given by a non-dimensional value of 0.04 , and the results with different cell-body aspect ratio are shown for the case with the constant hook rigidity of $\kappa / \tau=1$ and separation distance of $h / L=0.003$. The major axis is fixed as $c / L=0.15$, and the minor axis is changed within the values from $a / L=0.06$ to $a / L=0.15$. $(a, b)$ The time evolution of the cell-body inclination angle is plotted for a spheroidal $(a)$ and spherocylindrical $(b)$ cell body. $(c)$ The trajectories of the cell-body centre are shown for the spheroidal cell-body case. In the simulations of a spherocylindrical cell body, the simulations had to be terminated as the inclination angle exceeds the value of $\pi / 2$.

shape, and in this subsection, we numerically investigate the effects of the cell geometry on the stability of the vertical spinning behaviour.

In figure $11(a, b)$, the time evolution of the inclination angle $\theta$ is plotted for the simulation of a pusher bacterial swimmer with different aspect ratios of the cell-body geometry. We have fixed the parameter values, $h / L=0.003, \kappa / \tau=1$ and $c / L=0.15$, while the dynamics for the different values of $a / L$ has been examined from $a / L=0.06$ (elongated shape) to $a / L=0.15$ (sphere). The corresponding trajectories of the cell-body centre are also shown for the spheroidal case (figure 11c). These sets of the parameter values correspond to the stable upright spinning motion for a spherical cell body, but the spinning dynamics is found to be destabilized as the cell body becomes elongated in both cases of a spheroid and a spherocylinder. From figure 11(c), we have found the spherocylindrical cell body slightly enhances the upright motion rather than the spheroidal case. The spherocylinder simulation, however, has been terminated before the inclination angle exceeds the value of $\theta=\pi / 2$, because the problem becomes discontinuous at the configuration. When the flagellum is rotated backwards, the bacterial cell comes to an upright spinning position even for the 
elongated cell geometry (figure not shown), emphasizing the robust upright spinning dynamics for a puller flagellum in contrast to the pusher flagellar case, where the stable upright motion can be realized only in a limited range of parameter values.

\section{Simulation results II: repulsive and adhesive wall boundary}

In the previous sections, we have considered a constant separation distance between the cell body and the wall boundary. In this section, the free-swimming dynamics will be examined as biophysically more reasonable situations. We, however, need further physical assumptions on the cell-boundary interactions to proceed with the numerical simulation, and we consider the repulsive and adhesive interactions as introduced in $\S 2.3$.

\subsection{Repulsive boundary}

We start by considering that the wall boundary is purely repulsive in the vicinity of the boundary, and this situation can be achieved by neglecting adhesion effects, $k=0$. From (2.14), the wall repulsion can be characterized by two parameters, the force strength $g$, and the force length scale $\lambda$. With non-dimensionalizing these parameters using the units described in $\S 2.4$, we have the dimensionless force strength, $F_{a}=g T / \mu$, and inverse dimensionless length scale, $F_{b}=L / \lambda$. We consider a short-range strong repulsion, and we set $F_{a}=100$ since the force strength does not affect the swimmer behaviour if the value is sufficiently large (Ishimoto \& Gaffney 2016). However, as seen in $\S 4.1$, the force length scale, $F_{b}$, determines the separation distance, and the bacterial dynamics with different length scales $F_{b}$ has been examined.

In figure 12, we have plotted the time evolutions of the inclination and bending angles for the cell body of spherical, spheroidal and spherocylindrical geometries. The axes of the cell body are given by $c / L=0.1$ for a sphere and $c / L=0.15$ for a spheroid and spherocylinder, using the same minor axis of $a / L=0.1$. The numerical simulations have been performed with different $F_{b}$ but with the same initial angle $\theta_{\text {init }}=0.25 \pi$ and initial position $H / L=0.3$. The hook rigidity is fixed as $\kappa / \tau=1$ and a pusher flagellum has been considered. We will compare the dynamics between free swimming with a repulsive wall and confined swimming with a constant separation distance in the previous sections.

Until the cell body reaches the region in the vicinity of the boundary (nondimensional time of 20-40), the cell body slightly changes the inclination angle to follow the boundary, and this is purely due to hydrodynamic interactions (Shum et al. 2010; Ishimoto \& Gaffney 2017). Once the cell body experiences the repulsive force, the bacterial cell begins to stand upright, accompanied by the sudden increase of the bending angle, $\varphi$. If the force length scale is sufficiently small (large $F_{b}$ ), due to the torque by the misalignment between the cell body and flagellar orientations, the bacterial cell finally reaches the upright position, as seen in the confined swimming of $\S 4$.

In the simulations with a spherical cell body (figure 12a) and a short force length scale $\lambda$, the separation distance immediately converges to constant values, which reflects the force length scale. Thus the upright motion can be understood similarly to the confined swimming. However, in the case with the longer length scale of the force repulsion, the lubrication interactions are not sufficiently strong to sustain the cell body in a certain position and the cell finally follows the boundary $(\theta=\pi / 2)$ with a considerably larger separation distance $(H / L \approx 0.15)$ than that which the 

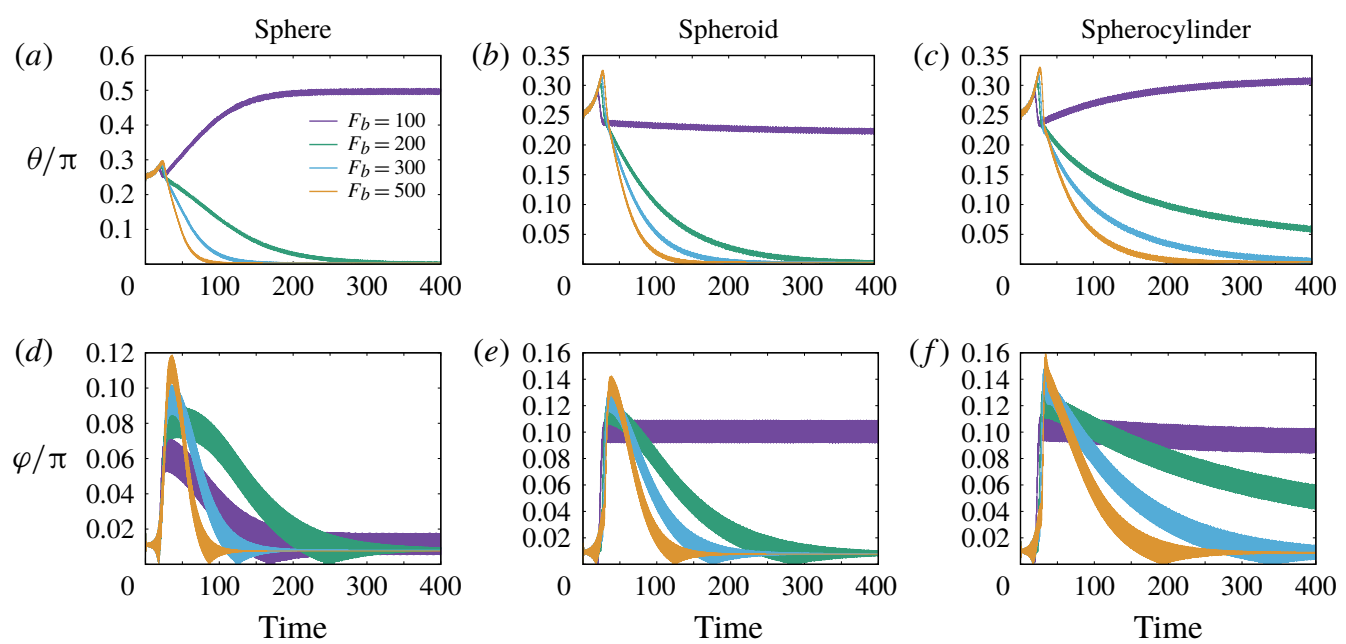

FIgURE 12. (Colour online) Time evolution of the cell-body inclination angle $\theta$ and the flagellar bending angle $\varphi$ in the free-swimming simulations of a model bacterium with different length scales of the wall repulsive force. The shape of the cell body is $(a)$ a sphere of radius $a / L=c / L=0.15,(b)$ a spheroid of axis lengths $(a / L, c / L)=(0.1,0.15)$, and $(c)$ a spherocylinder of axis lengths $(a / L, c / L)=(0.1,0.15)$. The inverse length scale of the wall force is varied within the range of $L / \lambda=F_{b}=\{100,200,300,500\}$, whereas constant values are used for the non-dimensional motor torque $(0.03)$, hook rigidity $\kappa / \tau=$ 1 , and non-dimensional strength of the wall repulsion $F_{a}=100$. The initial position of the cell-body centre is $H / L=0.3$ and the initial inclination angle is $\theta_{\text {init }}=0.25 \pi$.

repulsive force can affect $(H / L \lesssim 0.11)$. In this case, the boundary-following motion is stabilized purely by the hydrodynamic interactions as in the rigid hook case (Ishimoto \& Gaffney 2017).

The similar swimming behaviours are found in the spheroidal and spherocylindrical cases with large $F_{b}$ values, where the cell body gradually orients towards the vertical configuration due to the torque generated by the misalignment between the cell body and flagellar orientations (figure $12 b, c$ ). However, in the small $F_{b}$ case, the cell body is still oriented towards the boundary after a long time with nearly constant non-zero bending angles. This particular swimming dynamics can be characterized by a wallfollowing motion with continuous contacts to the boundary, and such a behaviour has been experimentally observed in $P$. aeruginosa bacterium and referred to as a horizontal spinning movement (de Anda et al. 2017).

\subsection{Adhesive boundary}

We then proceed to the free-swimming bacterial dynamics near an adhesive boundary to investigate the complex nature of the bacterial spinning top observed in a $P$. aeruginosa bacterial cell. We consider spring bonds between the cell body and the boundary, motivated by the nano-spring property of the pili adhesion (Beaussart et al. 2014).

The strength of the adhesion is characterized by the spring constant $k$ of the bond, and we use dimensionless values for the adhesion strength, defined as $K=k L T / \mu$. Other parameters regarding the creation and annihilation of adhesive bonds are given by $R_{a d} / L=0.01, R_{o n} / L=0.0101$ and $R_{o f f} / L=0.11$, following the previous studies 

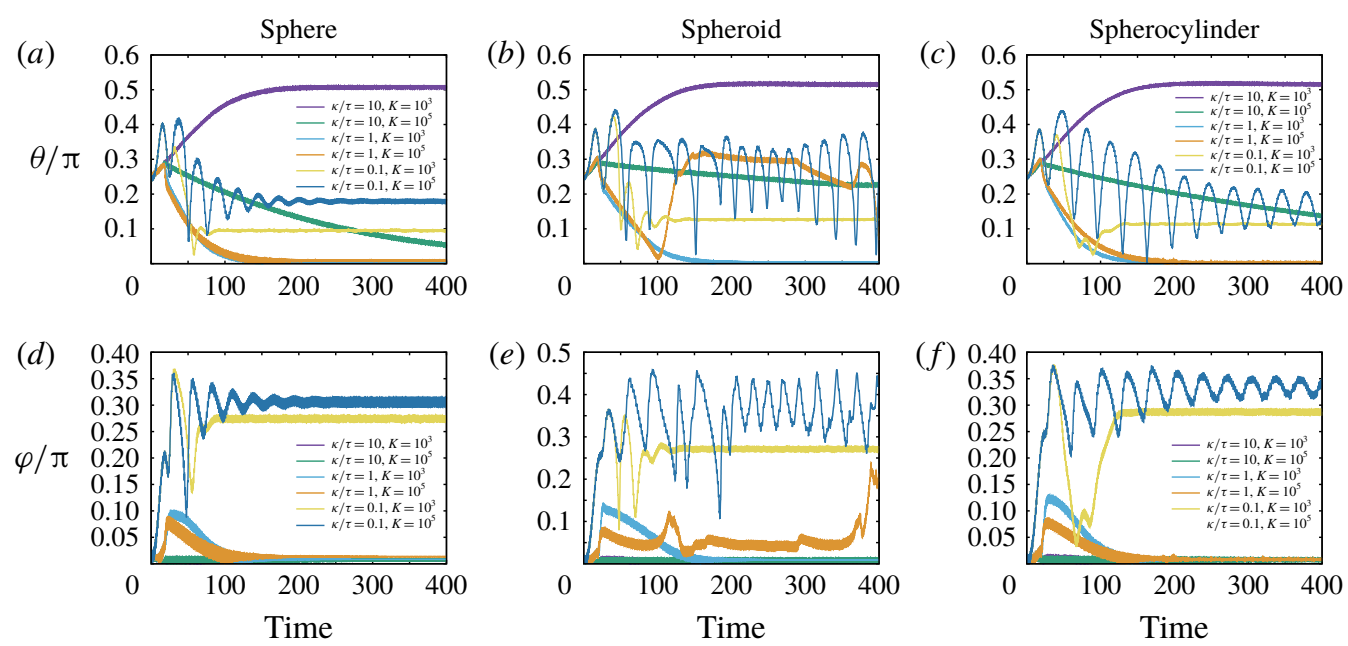

FIgURE 13. (Colour online) Time evolution of the cell-body inclination angle $\theta$ and the flagellar bending angle $\varphi$ in the simulations with adhesive boundary. The shape of the cell body is $(a)$ a sphere of radius $a / L=c / L=0.1,(b)$ a spheroid of axis lengths $(a / L, c / L)=$ $(0.1,0.15)$, and $(c)$ a spherocylinder of axis lengths $(a / L, c / L)=(0.1,0.15)$. The colours of the plots show the simulations with different values of hook rigidity $\kappa / \tau=\{10,1,0.1\}$ and adhesion strength $K=\left\{10^{3}, 10^{5}\right\}$. Constant values are used for the non-dimensional motor torque (0.03) and non-dimensional length scale and strength scale of the wall repulsion (100 and 300, respectively). The initial conditions are the same as in figure 12.

(Simons et al. 2014; Ishimoto \& Gaffney 2016). We also fix the values of the repulsive boundary force to be constant, $\left(F_{a}, F_{b}\right)=(100,300)$.

In figure 13, the angle behaviours for the three different geometries of the cell body are shown as in $\S 5.1$. We have examined bacterial dynamics with different values of hook rigidity $\kappa / \tau=\{10,1,0.1\}$ and adhesion strength $K=\left\{10^{3}, 10^{5}\right\}$.

With a spherical cell body, the bacterium has been stuck to the boundary, except for the case of $(\kappa / \tau, K)=\left(10,10^{3}\right)$, where the cell has exhibited a boundary-following motion (figure 13a). Remarkably, the strong adhesion $\left(K=10^{5}\right)$ enabled the cell to stand upright even with the rigid hook parameter $(\kappa / \tau=10)$. Note that the upright motion was not possible without adhesive interactions. With a flexible hook $(\kappa / \tau=0.1)$, the inclination and bending angles have converged to non-trivial values, and the cell has exhibited a top-like spinning motion around a certain perpendicular axis. To express this motion visually, we have traced two points on the cell body: one corresponds to the flagellar connection point, and the other is the opposite point with respect to the cell-body centre, following the presentations in de Anda et al. (2017) for comparison. The time evolutions of these points are shown in colour in figure $14(a)$.

In the spheroidal and spherocylindrical simulations (figure 13b,c,e,f), the cell body has eventually adhered to the boundary except for the cases with a rigid hook and weak adhesion value. The slight changes of the geometry have induced more complex behaviours such as non-steady behaviour with oscillatory angles, which emerge by the creation and annihilation of adhesive bonds. Example cell-body dynamics are shown in figure $14(b-d)$ for illustrations. In particular, with strong adhesion and flexible hook parameters, the angle values temporarily oscillate in an irregular manner, and 
(a) Sphere $(a / L=0.1)$
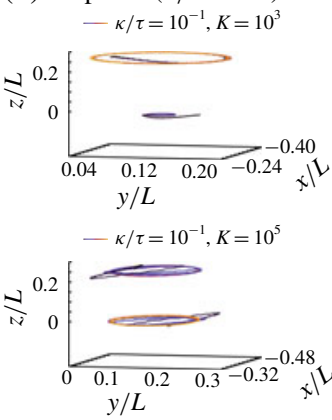

(b) Spheroid $(a / L=0.1)$
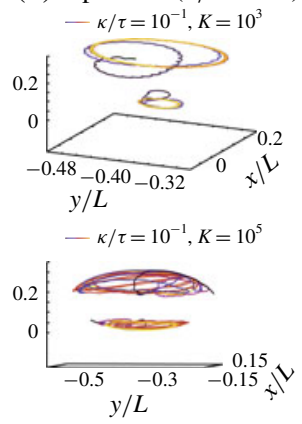

(c) Spherocylinder $(a / L=0.1)$
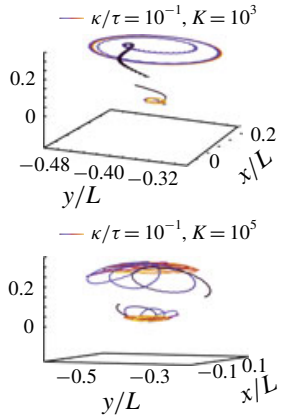

(d) Spheroid $(a / L=0.1)$
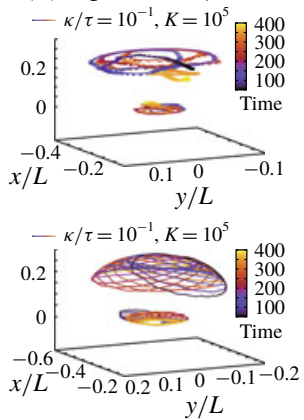

FIgURE 14. (Colour online) Example dynamics of the positions of the two pole ends of the cell body with different parameter values of adhesive strength, hook rigidity and cell-body geometry. The non-dimensional time is shown by the changes in colour. The constant parameters of the simulations are inherited from figure 13 . The geometry of the cell body is $(a)$ a sphere $a / L=c / L=0.1,(b)$ a spheroid of axis lengths $(a / L, c / L)=$ $(0.1,0.15),(c)$ a spherocylinder of axis lengths $(a / L, c / L)=(0.1,0.15)$, and $(d)$ a spheroid of axis lengths $(a / L, c / L)=(0.075,0.15)$.

the cell-body orbits become more complicated. Note that such unsteady dynamics of the bacterium have not been found in the simulations without adhesive interactions. Small differences of the aspect ratio are also found to generate further complex behaviours, as shown for the spheroidal cell-body case with $(a / L, c / L)=(0.075,0.15)$ in figure $14(d)$. These results suggest the rich diversity of the bacterial behaviour near an adhesive boundary, and the flexibility of the hook is found to be essential for the unsteady orbits.

\section{Discussions and conclusions}

We have numerically and theoretically investigated the near-boundary dynamics of a bacterial cell with a single flagellum that is connected to the cell body via a flexible hook, motivated by the recent observation of $P$. aeruginosa (de Anda et al. 2017), where the bacterial cells are reported to exhibit vertical spinning motions like a low-Reynolds-number spinning top. The primary aim of this study is to understand the hydrodynamics and stability of the bacterial spinning top behaviours, and we have approached this problem by combining a direct hydrodynamic numerical simulation via the boundary element method and a stability analysis based on the local hydrodynamic interactions.

First, we have focused on a spherical cell-body case with a constant separation distance from the wall boundary, establishing a theoretical framework to analyse the bacterial dynamics based on the lubrication theory for a spherical cell body near a boundary and the resistive force theory for axisymmetric flagellar propulsion. We have analysed the linear stability around the upright position and obtained a stability diagram that is much more complex than previously thought, as the stability depends on the direction of the motor torque, the hook rigidity and the lubrication-type hydrodynamic interactions near a boundary. The forwardly rotated flagellum that generates propulsive force towards the cell body, which we refer to as a pusher flagellum, can stabilize the upright spinning motion only in a limited range of hook rigidity. The backward rotation of the flagellum generates the force that pulls the 
cell body away, and we refer to this as a puller flagellum. In contrast to the pusher case, the puller flagellum is found to stabilize the upright motion in a wider range of parameter space. This remarkable difference of the stability originates from the large drag on the flagellum under a horizontal movement along the boundary. Moreover, in the rigid hook limit, we have found a critical flagellar length above which the vertical motion is unstable in the pusher flagellum case.

Further, we have demonstrated that the force and torque effects on the stability of the vertical spinning motion can be separately considered, which enables us to interpret the mechanical stability simply as the competition between the flagellar force and torque contributions. The pusher flagellar propulsive force contributes to destabilizing the vertical motion, whereas it is stabilized by the pulling propulsive force. The torque generation from the mechanical coupling between the cell-body rotation and the hook elasticity always contributes to the stabilization. Thus the stable vertical motion in the biologically relevant parameter region can be understood by the dominating stabilization effects of the flagellar torque contributions compared with the destabilization effects by the pushing propulsive force.

Now we consider simplified physical pictures that interpret the propulsive force and torque contributions in the linear stability of the vertical spinning motion. The flagellar propulsive force leads the translational motion of the cell body and thus the orientation of the cell body towards the upright position through the lubrication effect. The cell-body movement also generates the drag torque that orients the flagellum to follow the boundary. The longer flagellum experiences larger drag torque, and, in turn, the flagellar propulsive force destabilizes the vertical configuration for the parameters of typical flagellar morphology. The flagellar propulsive torque, on the other hand, generates the spinning motion of the cell body, which gives rise to a misalignment between the cell body and the flagellum orientations due to the drag force on the flagellum whose torque is balanced by the torque from the elastic hook. The orientation of the propulsive torque vector is then modulated by the misalignment so that the cell body rotates towards the vertical spinning configuration. For further clarification of these mechanical pictures, investigations of simple theoretical models are useful and will be reported elsewhere.

We then proceeded to full numerical simulations and found a notable agreement with the prediction by the local interaction theory in large parameter regions, supporting the intuitive understanding based on the local hydrodynamics. In particular, it is found that the spinning top behaviour can be maintained by the torque generation through misalignment of the orientations of the cell body and the flagellum, as suggested by the theoretical stability analysis. The torque generation by the misalignment was argued by the simple mathematical model in de Anda et al. (2017), where the cell body is anchored to the wall boundary and the mechanical stability was not analysed. The physical interpretation developed in this study, however, has clarified the further complicated mechanical competition between the destabilizing force contribution and the stabilizing torque generated by the misalignment.

Moreover, the simulation has revealed a more complex stability diagram, including intermediate stable angle and stable behaviours in very flexible parameter regions, where the flagellar bending angles are oscillatory in time, and the axisymmetric flagellar propulsion model would not be a feasible assumption.

The secondary aim of this study is to understand the rich diversity of the bacterial spinning top dynamics. We have started with the effects from the elongated shape of the bacterial cell body, and from the numerical simulation using a spheroidal and spherocylindrical geometry for the cell body, we have found that an elongated shape 
promotes the instability for the upright spinning motion and that the slight difference of the cell shape also affects the bacterial locomotion, which again highlights the subtle mechanical balance between the lubrication hydrodynamics and hook elasticity coupled with the cell locomotion.

The simulation results, therefore, suggest that upright motion may not be reasonable for the biologically relevant cell shape of the $P$. aeruginosa bacterium unless we consider non-hydrodynamic interactions between the cell and the wall boundary. In contrast to the pusher flagellum, the motility via the puller flagellum can reach the upright configuration in a broad range of the parameter values. Therefore, the puller flagellar cell would be easily able to escape from the boundary even after reaching the upright position, if the pulling force exceeds the attracting potential force. Thus, the change of the orientation of the motor torque could be very useful for a bacterial cell to control the boundary locomotion and boundary accumulation properties. Note, however, that the Pseudomonas bacteria utilize the pili for walking and twitching motility together with the flagellum-driven swimming, and both are considered to contribute to the surface detachment behaviour as essential mechanisms for the initial stage of biofilm formation (Conrad et al. 2011).

Finally, we have considered a free-swimming cell near a wall boundary with short-range interactions, modelling as a combination of the strong short-range repulsion and the adhesive forces represented by elastic spring bonds. The simulations without adhesion interactions are reduced to the purely repulsive boundary case, and we have reproduced the corresponding stability results obtained in the dynamics with a constant separation distance for the pusher flagellum cases. We have also found that with a non-spherical cell body the bacterium exhibits a horizontal spinning motion with continuous attachments to the boundary with a non-zero angle between the cell body and flagellar orientations. The addition of the adhesive interactions has enabled the bacterium cell to be stuck to the wall boundary and to exhibit more complex behaviours, including unstable orbits of a spinning top. Note, however, that, without adhesive interactions, such an unsteady dynamics has not been obtained. The characteristic time scale for the bacterial to stand upright is experimentally obtained as $\approx 0.2 \mathrm{~s}$. This corresponds to the non-dimensional value of $\approx 10$ in the current study and interestingly agrees with the dynamical time scale of the unstable orbit in the simulation results.

These qualitative and qualitative agreements in the varieties of complex behaviours suggest that the rich diversity of $P$. aeruginosa motility in experimental observations could be explained by the boundary adhesions and diverse bacterial morphology. However, we have not considered the detailed interactions through the pili, although the adhesive effects may capture some pili-boundary interactions. Furthermore, the simulation results suggest that the variety of cell morphologies, depending on the culture medium and cell life cycle, would affect the bacterial surface behaviours. We also note that the motor torque is assumed to be constant in this study, but the direction of the motor rotation is considered to be regulated in a sophisticated way with sensing the surrounding environments (Persat 2017; Conrad \& Poling-Skutvik 2018). For the detailed mechanism of the complex behaviours of bacterial surface locomotion, further experimental and theoretical studies are required. Thus, precise simulations to reproduce the $P$. aeruginosa boundary locomotions are beyond the current study.

Nonetheless, we have successfully established a theoretical framework on the stability analysis for this complicated hydrodynamic phenomenon. This theoretical framework should be applicable to other physical situations regarding the elasto- 
hydrodynamic stability problems of bacterial locomotion such as in an external flow (Shen et al. 2012; Conrad \& Poling-Skutvik 2018).

We have found that a simple flagellar pushing force cannot stabilize the vertical motion, suggesting the necessity of a different mechanism for a spinning Thiovulum bacterium (Petroff et al. 2015; Petroff \& Libchaber 2018). As noted in Petroff et al. (2015), T. majus is a peritrichous bacterium with numerous short flagella (Boer, Riviere \& Houwink 1961) and the multi-flagellarity may be required to understand its underlying mechanism. The elastohydrodynamic stability analysis of a multi-flagellated bacterium (Ishimoto \& Lauga 2019) suggests that the vertical spinning mode is the most unstable when the flagellum is sufficiently short, but detailed theoretical analysis has not yet been done in the presence of the wall boundary. Further, the biased rotation direction of $T$. majus could be understood by the symmetry being broken by the flagellar chirality and inhomogeneous propulsion due to the presence of a wall, as found in the anticlockwise rotation of a sperm-egg cluster in mice in vitro fertilization (Ishimoto, Ikawa \& Okabe 2017). Similarly, the elastohydrodynamics in the swimming of multi-flagellated bacteria would provide a better understanding of the boundary-following swimming of E. coli. It is, however, suggested from our results that the lack of vertical motion in E. coli bacteria is due to the coherent flagella bundle of a swimming E. coli cell, as this bundle structure can be regarded as a very rigid hook, as is often assumed (Lauga et al. 2006). Nonetheless, further studies are required to conclude the mechanisms of these surface behaviours of peritrichous bacteria.

In summary, we have theoretically and numerically investigated the bacterial dynamics near a wall boundary and found that the vertical spinning motion like a bacterial spinning top is enabled by the mechanical competition between the flagellar force destabilization and stabilization due to the coupling of the hook elasticity with cell rotational motility. We also found that the non-spherical geometry of the cell body and boundary adhesive interactions affect the bacterial dynamics, leading to complex behaviours, including horizontal spinning and unstable vertical spinning motions, as experimentally observed. These results demonstrate the mechanical nature of the behaviours in rich diversity, and could contribute to our deeper understanding of the bacterial surface motility and biofilm formation.

\section{Acknowledgements}

K.I. is supported by JSPS-KAKENHI for Young Researchers (18K13456), JSPS Overseas Research Fellowship, and MEXT Leading Initiative for Excellent Young Researchers (LEADER). Elements of the simulations have been performed using the cluster computing system within the Research Institute for Mathematical Sciences (RIMS), and the Institute for Information Management and Communication (IIMC), Kyoto University.

\section{Appendix A. Hydrodynamic drag coefficients used in the local interaction theory}

In this appendix, we summarize the values for the drag coefficients used in the local interaction theory. The resistive matrix for a sphere near an infinite wall boundary is introduced in (3.1), whose components are

$$
\boldsymbol{c}_{T T}=\left(\begin{array}{ccc}
C_{D} & 0 & 0 \\
0 & C_{D} & 0 \\
0 & 0 & C_{D_{z}}
\end{array}\right), \quad \boldsymbol{c}_{T R}=\left(\begin{array}{ccc}
0 & -C_{S} & 0 \\
C_{S} & 0 & 0 \\
0 & 0 & 0
\end{array}\right),
$$




$$
\boldsymbol{c}_{R T}=\left(\begin{array}{ccc}
0 & -C_{S}^{\prime} & 0 \\
C_{S}^{\prime} & 0 & 0 \\
0 & 0 & 0
\end{array}\right), \quad \boldsymbol{c}_{R R}=\left(\begin{array}{ccc}
C_{R} & 0 & 0 \\
0 & C_{R} & 0 \\
0 & 0 & C_{R_{z}}
\end{array}\right),
$$

where the detailed expressions are asymptotically given by (Goldmann et al. 1967)

$$
\begin{array}{cl}
C_{D}=-6 \pi \mu a\left[\frac{8}{15} \log \left(\frac{a}{h}\right)+0.9588\right], & C_{R}=-8 \pi \mu a^{3}\left[\frac{2}{5} \log \left(\frac{a}{h}\right)+0.3817\right], \\
C_{S}=-6 \pi \mu a\left[\frac{2}{15} \log \left(\frac{a}{h}\right)-0.2526\right], & C_{S}^{\prime}=-8 \pi \mu a^{3}\left[\frac{1}{10} \log \left(\frac{a}{h}\right)-0.1895\right], \\
C_{R_{z}}=-8 \pi \mu a^{3}, & C_{D_{z}}=-6 \pi \mu a\left(\frac{a}{h}\right) .
\end{array}
$$

We approximate the flagellum as a simple left-handed helix with effective length $L_{e}$, helix radius $A$ and pitch $2 \pi / k$. The flagellar drag coefficients are obtained by the resistive force theory (Lauga et al. 2006),

$$
\begin{gathered}
k_{C}=-2 C_{T} L_{e}\left(1+3 \epsilon^{2} / 4\right) / \sqrt{1+\epsilon^{2}}, \\
K_{C}=-C_{T} L_{e}\left(1+2 \epsilon^{2}\right) / \sqrt{1+\epsilon^{2}}, \\
k_{T}=-C_{T} L_{e}^{2}\left(1+3 \epsilon^{2} / 4\right) / \sqrt{1+\epsilon^{2}}, \\
K_{T}=-C_{T} L_{e} b^{2} k / \sqrt{1+\epsilon^{2}}, \\
k_{D}=(1 / 2) C_{T} L_{e} b^{2} k / \sqrt{1+\epsilon^{2}}, \\
k_{F}=-(2 / 3) C_{T} L_{e}^{3}\left(1+3 \epsilon^{2} / 4\right) / \sqrt{1+\epsilon^{2}}, \\
K_{F}=-2 C_{T} L_{e} b^{2}\left(1+\epsilon^{2} / 2\right) / \sqrt{1+\epsilon^{2}},
\end{gathered}
$$

where $\epsilon=A k$ and the coefficient prefactor $C_{T}$ is $C_{T}=2 \pi \mu / \log \left(2 L_{e} / d\right)$ with $d$ being the flagellar radius.

\section{REFERENCES}

de Anda, J., Lee, E. Y., Lee, C. K., Bennet, R. R., Ji, X., Soltani, S., Harrison, M. C., BAKer, A. E., Luo, Y., Chou, T. et al. 2017 High-speed '4d' computational microscopy of bacterial surface motility. ACS Nano 11, 9340-9351.

Beaussart, A., Baker, A. E., Kuchma, S. L., El-Kirat-Chatel, S., O’Toole, G. A. \& DUfreñe, Y. F. 2014 Nanoscale adhesion forces of Pseudomonas aeruginosa type IV pili. ACS Nano 10, 10723-10733.

Bennett, R. R., Lee, C. K., Anda, J. D., Nealson, K. H., Yildiz, F. H., O’Toole, G. A., Wong, G. C. L. \& Golestanian, R. 2016 Species-dependent hydrodynamics of flagellumtethered bacteria in early biofilm development. J. R. Soc. Interface 13, 20150966.

Bianchi, S., Saglimbeni, F. \& Di Leonardo, R. 2017 Holographic imaging reveals the mechanisms of wall entrapment in swimming bacteria. Phys. Rev. X 7, 011010.

Blake, J. R. 1971 A note on the image system for a stokeslet in a no-slip boundary. Proc. Camb. Phil. Soc. 70, 303-310.

Boer, W. E. D., Riviere, J. W. M. L. \& Houwink, A. L. 1961 Observations on the morphology of Thiovulum majus Hinze. Antonie Leeuwenhoek 27, 447-456. 
BraAms, C. M. 1952 On the influence of friction on the motion of a top. Physica 18, 503-514.

Chen, Y., Busscher, H. J., van Der MeI, H. C. \& Norde, W. 2011 Statistical analysis of longand short-range forces involved in bacterial adhesion to substratum surfaces as measured using atomic force microscopy. Appl. Environ. Microbiol. 77, 5065-5070.

Conrad, J. C., Gibiansky, M. L., Jin, F., Gordon, V. D., Motto, D. A., Mathewson, M. A., Stopka, W. G., Zelasko, D. C., Shrout, J. D. \& Wong, G. C. L. 2011 Flagella and pili-mediated near-surface single-cell motility mechanisms in P. aeruginosa. Biophys. $J$. 100, 1608-1616.

Conrad, J. C. \& Poling-Skutvik, R. 2018 Confined flow: consequences and implications for bacteria and biofilms. Annu. Rev. Chem. Biomol. Engng 9, 175-200.

Dasgupta, N., Arora, S. K. \& Ramphal, R. 2004 The flagellar system of Pseudomonas aeruginosa. In Pseudomonas (ed. J. L. Ramos), pp. 675-698. Springer.

Drescher, K., Dunkel, J., Cisneros, L. H., Ganguly, S. \& Goldstein, R. E. 2011 Fluid dynamics and noise in bacterial cell-cell and cell-surface scattering. Proc. Natl Acad. Sci. USA 108, 10940-10945.

Drescher, K., Dunkel, J., Nadell, C. D., Van Teeffelen, S., Grnja, I., Wingreen, N. S., Stone, H. A. \& BASSLER, B. L. 2016 Architectural transitions in Vibrio cholerae biofilms at single-cell resolution. Proc. Natl Acad. Sci. USA 113, E2066-E2072.

Goldmann, A. J., Cox, R. G. \& BRenner, H. 1967 Slow viscous motion of a sphere parallel to a plane wall - II Couette flow. Chem. Engng Sci. 22, 633-660.

Higdon, J. J. L. 1979 The hydrodynamics of flagellar propulsion: helical waves. J. Fluid Mech. 94, 331-357.

Ishikawa, T. \& Pedley, T. J. 2007 Diffusion of swimming model micro-organisms in a semi-dilute suspension. J. Fluid Mech. 588, 437-462.

Ishimoto, K. \& GaffNey, E. A. 2013 Squirmer dynamics near a boundary. Phys. Rev. E 88, 062702.

Ishimoto, K. \& GAFFney, E. A. 2014 A study of spermatozoan swimming stability near a surface. J. Theor. Biol. 360, 187-199.

Ishimoto, K. \& GAFFNEY, E. A. 2016 Mechanical tuning of mammalian sperm behaviour by hyperactivation, rheology and substrate adhesion: a numerical exploration. J. R. Soc. Interface 13, 20160633.

Ishimoto, K. \& GAFFney, E. A. 2017 Boundary element methods for particles and microswimmers in a linear viscoelastic fluid. J. Fluid Mech. 831, 228-251.

Ishimoto, K., IKAWA, M. \& OKABE, M. 2017 The mechanics clarifying counterclockwise rotation of most IVF eggs in mice. Sci. Rep. 7, 43456.

Ishimoto, K. \& LAUGA, E. 2019 The N-flagella problem: elastohydrodynamic motility transition of multi-flagellated bacteria. Proc. R. Soc. Lond. A 475, 20180690.

JabBarzadeh, M. \& FU, H. C. 2018 Dynamic instability in the hook-flagellum system that triggers bacterial flicks. Phys. Rev. E 97, 012402.

Klein, J. D., Clapp, A. R. \& Dickinson, R. B. 2003 Direct measurement of interaction forces between a single bacterium and a flat plate. J. Colloid Interface Sci. 261, 379-385.

Lauga, E. 2016 Bacterial hydrodynamics. Annu. Rev. Fluid Mech. 48, 105-130.

Lauga, E., Diluzio, W. R., Whiteside, G. M. \& Stone, H. A. 2006 Swimming in circles: motion of bacteria near solid boundaries. Biophys. J. 90, 400-412.

Lauga, E. \& Powers, T. R. 2009 The hydrodynamics of swimming microorganisms. Rep. Prog. Phys. 72, 096601.

Maier, B. \& Wong, G. C. L. 2015 How bacteria use type IV pili machinery on surfaces. Trends Microbiol. 23, 775-788.

Moffatt, H. K. \& Shimomura, Y. 2002 Spinning eggs - a paradox resolved. Nature 416, 385-386.

Moffatt, H. K., Shimomura, Y. \& BRAnicki, M. 2004 Dynamics of an axisymmetric body spinning on a horizontal surface. I. Stability and the gyroscopic approximation. Proc. R. Soc. Lond. A 460, 3643-3672.

Nguyen, F. T. M. \& GrahaM, M. D. 2017 Bucking instabilities and complex trajectories in a simple model of uniflagellar bacteria. Biophys. J. 112, 1010-1022. 
NGuYen, F. T. M. \& Graham, M. D. 2018 Impacts of multiflagellarity on stability and speed of bacterial locomotion. Phys. Rev. E 98, 042419.

Perni, S., Preedy, E. C. \& Prokopovich, P. 2014 Success and failure of colloidal approaches in adhesion of microorganisms to surfaces. Adv. Colloid Interface Sci. 206, 265-274.

Persat, A. 2017 Bacterial mechanotransduction. Curr. Opin. Microbiol. 36, 1-6.

Persat, A., Nadell, C. D., Kim, M. K., Ingremeau, F., Siryaporn, A., Drescher, K., Wingreen, N. S., BAssler, B. L., Gitai, Z. \& Stone, H. A. 2015 The mechanical world of bacteria. Cell 161, 988-997.

Petroff, A. P. \& Libchaber, A. 2018 Nucleation of rotating crystals by Thiovulum majus bacteria. New J. Phys. 20, 015007.

Petroff, A. P., Wu, X.-L. \& Libchaber, A. 2015 Fast-moving bacteria self-organize into active two-dimensional crystals of rotating cells. Phys. Rev. Lett. 114, 158102.

Pozrikidis, C. 2002 A Practical Guide to Boundary Element Methods with the Software Library BEMLIB. CRC Press.

Riley, E. E., DAS, D. \& LAUga, E. 2018 Swimming of peritrichous bacteria is enabled by an elastohydrodynamic instability. Sci. Rep. 8, 10728.

Shen, Y., Siryaporn, A., Lecuyer, S., Gitai, Z. \& Stone, H. A. 2012 Flow directs surfaceattached bacteria to twitch upstream. Biophys. J. 103, 146-151.

Shimogonya, Y., Sawano, Y., Wakabe, H., Inoue, Y., IshiJima, A. \& IshiKawa, T. 2015 Torque-induced precession of bacterial flagella. Sci. Rep. 5, 18488.

Shum, H. \& GAFFneY, E. A. 2012 The effects of flagellar hook compliance on motility of monotrichous bacteria: a modeling study. Phys. Fluids 24, 061901.

Shum, H., Gaffney, E. A. \& SMith, D. J. 2010 Modelling bacterial behaviour close to a no-slip plane boundary: the influence of bacterial geometry. Proc. R. Soc. Lond. A 486, 1725-1748.

Simons, J., Olson, S., Cortez, R. \& FAUCI, L. 2014 The dynamics of sperm detachment from epithelium in a coupled fluid-biochemical model of hyperactivated motility. J. Theor. Biol. 354, 81-94.

Smith, D. J. 2018 A nearest-neighbour discretisation of the regularized stokeslet boundary integral equation. J. Comput. Phys. 358, 88-102.

Smith, D. J., Gaffney, E. A., Blake, J. R. \& Kirkman-Brown, J. C. 2009 Human sperm accumulation near surfaces: a simulation study. J. Fluid Mech. 621, 289-320.

Son, K., Guasto, J. S. \& Stocker, R. 2013 Bacteria can exploit a flagellar buckling instability to change direction. Nat. Phys. 9, 494-498.

Spagnolie, S. E. \& LAUGA, E. 2012 Hydrodynamics of self-propulsion near a boundary: prediction and accuracy of far-field approximations. J. Fluid Mech. 700, 105-147.

WAKIYA, S. 1975 Application of bipolar coordinates to the two-dimensional creeping motion of a liquid. II. Some problems for two circular cylinders in viscous fluid. J. Phys. Soc. Japan 39, 1603-1607. 